\title{
SEDIGISM: Structure, excitation, and dynamics of the inner Galactic interstellar medium $\star, \star \star$
}

\author{
F. Schuller ${ }^{1,2}$, T. Csengeri ${ }^{1}$, J. S. Urquhart ${ }^{1,3}$, A. Duarte-Cabral ${ }^{4}$, P. J. Barnes ${ }^{5,6}$, A. Giannetti ${ }^{1}$, A. K. Hernandez ${ }^{7}$,
} S. Leurini ${ }^{1}$, M. Mattern ${ }^{1}$, S.-N. X. Medina ${ }^{1}$, C. Agurto $^{2}$, F. Azagra ${ }^{2}$, L. D. Anderson ${ }^{8}$, M. T. Beltrán ${ }^{9}$, H. Beuther ${ }^{10}$, S. Bontemps ${ }^{11}$, L. Bronfman ${ }^{12}$, C. L. Dobbs ${ }^{4}$, M. Dumke ${ }^{2}$, R. Finger ${ }^{12}$, A. Ginsburg ${ }^{13}$, E. Gonzalez ${ }^{2}$, T. Henning ${ }^{10}$, J. Kauffmann ${ }^{1}$, F. Mac-Auliffe ${ }^{2}$, K. M. Menten ${ }^{1}$, F. M. Montenegro-Montes ${ }^{2}$, T. J. T. Moore ${ }^{14}$, E. Muller ${ }^{15}$, R. Parra ${ }^{2}$, J.-P. Perez-Beaupuits ${ }^{2}$, A. Pettitt ${ }^{16}$, D. Russeil ${ }^{17}$, Á. Sánchez-Monge ${ }^{18}$, P. Schilke ${ }^{18}$, E. Schisano ${ }^{19}$, S. Suri ${ }^{18}$, L. Testi ${ }^{13}$, K. Torstensson ${ }^{2}$, P. Venegas ${ }^{2}$, K. Wang ${ }^{13}$, M. Wienen ${ }^{1}$, F. Wyrowski ${ }^{1}$, and A. Zavagno ${ }^{17}$

1 Max-Planck-Institut für Radioastronomie, Auf dem Hügel 69, 53121 Bonn, Germany e-mail: schuller@mpifr-bonn.mpg . de

2 European Southern Observatory, Alonso de Cordova 3107, Casilla 19001 Santiago 19, Chile

3 Centre for Astrophysics and Planetary Science, University of Kent, Canterbury, CT2 7NH, UK

${ }^{4}$ School of Physics, University of Exeter, Stocker Road, Exeter, EX4 4QL, UK

5 Astronomy Department, University of Florida, PO Box 112055, Gainesville, FL 32611, USA

6 School of Science and Technology, University of New England, NSW 2351 Armidale, Australia

7 Astronomy Department, University of Wisconsin, 475 North Charter St., Madison, WI 53706, USA

8 West Virginia University, Department of Physics \& Astronomy, PO Box 6315, Morgantown, WV 26506, USA

9 Osservatorio Astrofisico di Arcetri, Largo Enrico Fermi 5, 50125 Firenze, Italy

10 Max-Planck-Institut für Astronomie, Königstuhl 17, 69117 Heidelberg, Germany

11 Université Bordeaux, LAB, CNRS, UMR 5804, 33270 Floirac, France

12 Departamento de Astronomía, Universidad de Chile, Casilla 36-D, Santiago, Chile

13 European Southern Observatory, Karl-Schwarzschild-Str. 2, 85748 Garching bei München, Germany

14 Astrophysics Research Institute, Liverpool John Moores University, 146 Brownlow Hill, Liverpool, L3 5RF, UK

15 National Astronomical Observatory of Japan, Chile Observatory, 2-21-1 Osawa, Mitaka, 181-8588 Tokyo, Japan

16 Department of Physics, Faculty of Science, Hokkaido University, 060-0810 Sapporo, Japan

17 Laboratoire d'Astrophysique de Marseille, Aix Marseille Université, CNRS, UMR 7326, 13388 Marseille, France

18 I. Physikalisches Institut, Universität zu Köln, Zülpicher Str. 77, 50937 Köln, Germany

19 Istituto di Astrofisica e Planetologia Spaziali, INAF, via Fosso del Cavaliere 100, 00133 Roma, Italy

Received 13 May 2016 / Accepted 26 December 2016

\begin{abstract}
Context. The origin and life-cycle of molecular clouds are still poorly constrained, despite their importance for understanding the evolution of the interstellar medium. Many large-scale surveys of the Galactic plane have been conducted recently, allowing for rapid progress in this field. Nevertheless, a sub-arcminute resolution global view of the large-scale distribution of molecular gas, from the diffuse medium to dense clouds and clumps, and of their relationship to the spiral structure, is still missing.

Aims. We have carried out a systematic, homogeneous, spectroscopic survey of the inner Galactic plane, in order to complement the many continuum Galactic surveys available with crucial distance and gas-kinematic information. Our aim is to combine this data set with recent infrared to sub-millimetre surveys at similar angular resolutions.

Methods. The SEDIGISM survey covers $78 \mathrm{deg}^{2}$ of the inner Galaxy $\left(-60^{\circ} \leq \ell \leq 18^{\circ},|b| \leq 0.5^{\circ}\right)$ in the $J=2-1$ rotational transition of ${ }^{13} \mathrm{CO}$. This isotopologue of CO is less abundant than ${ }^{12} \mathrm{CO}$ by factors up to 100 . Therefore, its emission has low to moderate optical depths, and higher critical density, making it an ideal tracer of the cold, dense interstellar medium. The data have been observed with the SHFI single-pixel instrument at APEX. The observational setup covers the ${ }^{13} \mathrm{CO}(2-1)$ and $\mathrm{C}^{18} \mathrm{O}(2-1)$ lines, plus several transitions from other molecules.

Results. The observations have been completed. Data reduction is in progress, and the final data products will be made available in the near future. Here we give a detailed description of the survey and the dedicated data reduction pipeline. To illustrate the scientific potential of this survey, preliminary results based on a science demonstration field covering $-20^{\circ} \leq \ell \leq-18.5^{\circ}$ are presented. Analysis of the ${ }^{13} \mathrm{CO}(2-1)$ data in this field reveals compact clumps, diffuse clouds, and filamentary structures at a range of heliocentric distances. By combining our data with data in the (1-0) transition of CO isotopologues from the ThrUMMS survey, we are able to compute a 3D realization of the excitation temperature and optical depth in the interstellar medium. Ultimately, this survey will provide a detailed, global view of the inner Galactic interstellar medium at an unprecedented angular resolution of $\sim 30^{\prime \prime}$.
\end{abstract}

Key words. surveys - Galaxy: structure - radio lines: ISM - ISM: clouds - ISM: structure

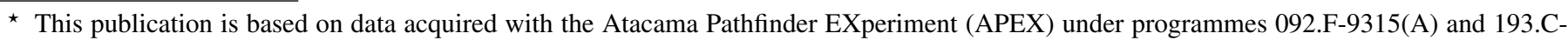
0584(A). APEX is a collaboration between the Max-Planck-Institut für Radioastronomie, the European Southern Observatory, and the Onsala Space Observatory.

$\star \star$ Full Table 5 and Table A.1 are only available at the CDS via anonymous ftp to cdsarc.u-strasbg.fr $(130.79 .128 .5)$ or via http://cdsarc.u-strasbg.fr/viz-bin/qcat?J/A+A/601/A124
} 


\section{Introduction}

Despite being our home, the global structure of the Milky Way is still poorly constrained. There is substantial ongoing effort to simulate our Galaxy's spiral arms and bar potentials (Mulder \& Liem 1986; Khoperskov et al. 2013; Pettitt et al. 2014, 2015, among others). However, only observations of the entire Galaxy can provide constraints for the gas distribution required for such models.

Continuum surveys from the infrared to the millimetre, such as GLIMPSE (Benjamin et al. 2003), MIPSGAL (Carey et al. 2009), WISE (Wright et al. 2010), Hi-GAL (Molinari et al. 2010), ATLASGAL (Schuller et al. 2009), and the BGPS (Aguirre et al. 2011), are sensitive to thermal emission from dust grains, associated with dense interstellar gas. Radio-continuum surveys give complementary views of the heated, ionised gas (e.g. CORNISH, Hoare et al. 2012 and THOR, Bihr et al. 2016). These surveys provide catalogues with several thousand compact objects (e.g. ATLASGAL; Csengeri et al. 2014; Urquhart et al. 2014a), up to over a million sources for infrared surveys (e.g. MIPSGAL, Gutermuth \& Heyer 2015), revealing the recent and ongoing star-formation activity throughout the inner Galaxy. In addition, mid-infrared surveys reveal thousands of infrared dark clouds (IRDC, e.g. Peretto \& Fuller 2009), which are seen in extinction against the bright Galactic background emission.

Spectroscopic follow-up observations are essential to measure radial velocities $\left(v_{\mathrm{lsr}}\right)$ of the molecular clouds and clumps detected in far-IR and (sub-)mm surveys, and to determine their kinematic distances; to constrain their virial mass and gravitational state (bound or unbound); and to constrain the gas excitation, chemical abundances, and turbulence, all of which are key parameters in theoretical models of star formation. Currently available molecular surveys of the inner Galaxy include (for a more complete list, see Heyer \& Dame 2015): the Galactic Ring Survey (GRS) in ${ }^{13} \mathrm{CO}(1-0)$ (Jackson et al. 2006), which covers a Galactic longitude range of $\sim 17-55^{\circ}$; the $\mathrm{HOPS} \mathrm{NH}_{3}$ and $\mathrm{H}_{2} \mathrm{O}$ maser survey (Walsh et al. 2011), which traces dense gas components over $-70^{\circ}<\ell<30^{\circ}$; the CHaMP survey that covers the Carina tangent $\left(280^{\circ}<\ell<300^{\circ}\right.$; Barnes et al. 2011, 2016) in the $J=1-0$ transitions of ${ }^{12} \mathrm{CO},{ }^{13} \mathrm{CO}, \mathrm{C}^{18} \mathrm{O}$, and $\mathrm{HCO}^{+}$; COHRS (Dempsey et al. 2013), covering $10^{\circ}<\ell<55^{\circ}$ in ${ }^{12} \mathrm{CO}(3-2)$; and CHIMPS (Rigby et al. 2016), covering $28^{\circ}<$ $\ell<46^{\circ}$ in ${ }^{13} \mathrm{CO}(3-2)$ and $\mathrm{C}^{18} \mathrm{O}(3-2)$. Most of these surveys cover relatively small $\left(\sim 20-40^{\circ}\right)$ ranges in Galactic longitude.

Although they provide a global view of the Galaxy, the relatively low resolution ( $\sim 1 / 8$ degree) and high optical depth of the ${ }^{12} \mathrm{CO}(1-0)$ surveys by Bronfman et al. (1988) and Dame et al. (2001) limit our understanding of the dynamical structure of the inner Galaxy. Several ongoing surveys will ultimately cover large pieces of the Galaxy in $\mathrm{CO}(1-0)$ and isotopologues at high angular resolution: the complete and public ThrUMMS (Threemm Ultimate Mopra Milky Way Survey, Barnes et al. 2015) covers $-60^{\circ}<\ell<0^{\circ},|b|<1^{\circ}$ at $72^{\prime \prime}$ resolution; the ongoing Mopra Southern Galactic Plane CO survey (Burton et al. 2013; Braiding et al. 2015) has so far covered $10^{\circ} \times 1^{\circ}$, but is envisaged to cover $90^{\circ} \times 1^{\circ}$, with an angular resolution of $36^{\prime \prime}$; and the Milky Way Imaging Scroll Painting project (MWISP ${ }^{1}$ ), ongoing since 2011 , plans to cover $-10.25^{\circ} \leq \ell \leq 250.25^{\circ}$ over $|b| \leq$ $5.25^{\circ}$, with a $52^{\prime \prime}$ resolution.

To provide an even higher angular-resolution view of the inner Galaxy in mostly optically thin gas tracers, we have undertaken the structure, excitation, and dynamics of the inner Galactic interstellar medium (SEDIGISM) survey. This survey covers

http://wWW . radioast. nsdc. cn/mwisp.php

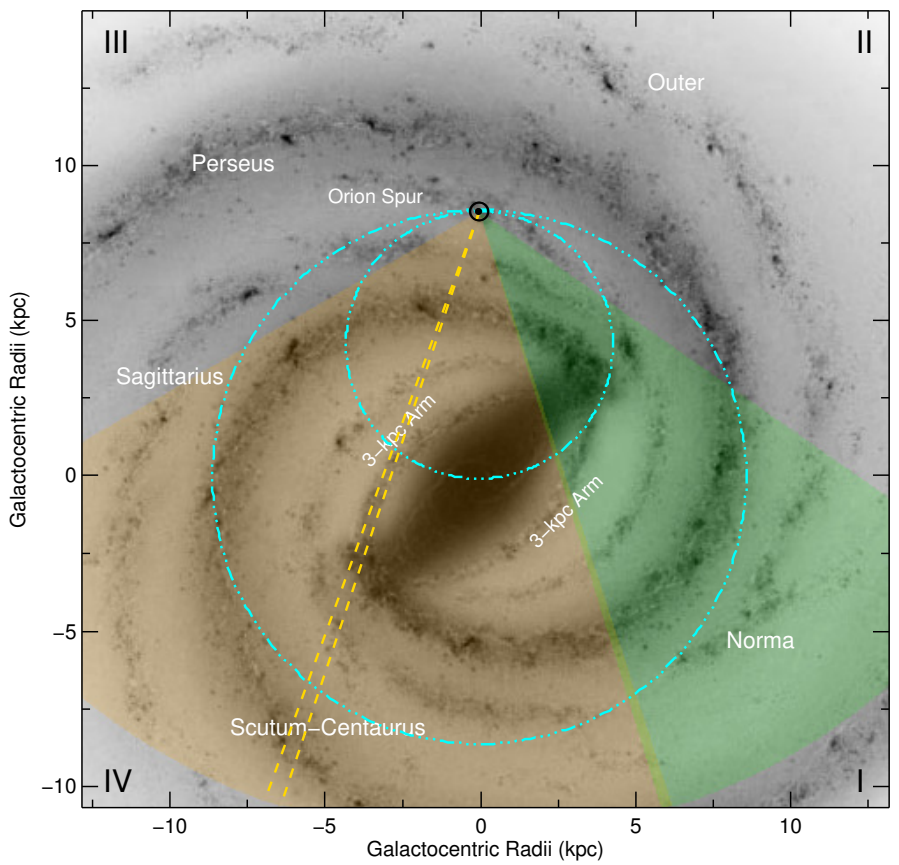

Fig. 1. Coverage of the SEDIGISM and GRS surveys, shown respectively in orange and green shading, overlaid on the top down image of the Milky Way produced by Robert Hurt of the Spitzer Science Center in consultation with Robert Benjamin (see Churchwell et al. 2009, for more details). The large and small cyan circles indicate the Solar Circle and the position of the tangent points (maximum radial velocity), respectively, while the dashed yellow lines demarcate the region selected as the science demonstration field (see Sect. 2). The position of the Sun is indicated by the $\odot$ symbol.

$78 \mathrm{deg}^{2}$ of the southern Galactic plane: $-60^{\circ} \leq \ell \leq 18^{\circ}$, with $|b| \leq$ $0.5^{\circ}$, at $30^{\prime \prime}$ resolution. This longitude range has been selected to provide complementary coverage to the GRS survey (see Fig. 1 for coverage map). The prime target of the SEDIGISM survey are the ${ }^{13} \mathrm{CO}(2-1)$ and $\mathrm{C}^{18} \mathrm{O}(2-1)$ transitions, which are usually optically thin in the Galactic instertellar medium (ISM). Therefore, they are well suited to trace the dense molecular gas. Thus, this survey provides fundamental information to constrain the Galactic structure, in particular, the number and position of spiral arms. It also provides crucial $v_{\mathrm{lsr}}$ measurements, allowing kinematic distances to be estimated, thus serving as an excellent resource for continuum surveys at comparable resolutions, such as ATLASGAL and Hi-GAL.

The ubiquitous presence of filaments in the Galactic ISM has been recognised with Herschel (e.g. Molinari et al. 2010; André et al. 2010) in both star-forming and quiescent clouds. Filaments play a pivotal role in the formation of stars, as it is within them that instabilities develop, leading to the formation of clumps and cores (e.g. Federrath 2016, Smith et al. 2016). In some more extreme cases, filamentary networks are thought to serve as channels for feeding mass onto protostellar cores in the early stages of star formation, providing the material needed for the formation of high-mass stars (e.g. Schneider et al. 2010; Peretto et al. 2013). Despite their importance, the exact formation mechanism of these structures is not yet fully understood. While some theories suggest that shocks in the ISM are responsible for forming filaments, either through the classical turbulent motions of gas (McKee \& Ostriker 2007; Federrath 2016), or through more extreme shocks from larger-scale converging flows (e.g. Heitsch et al. 2008), other theories suggest that magnetic fields play an important role in both forming and shaping 


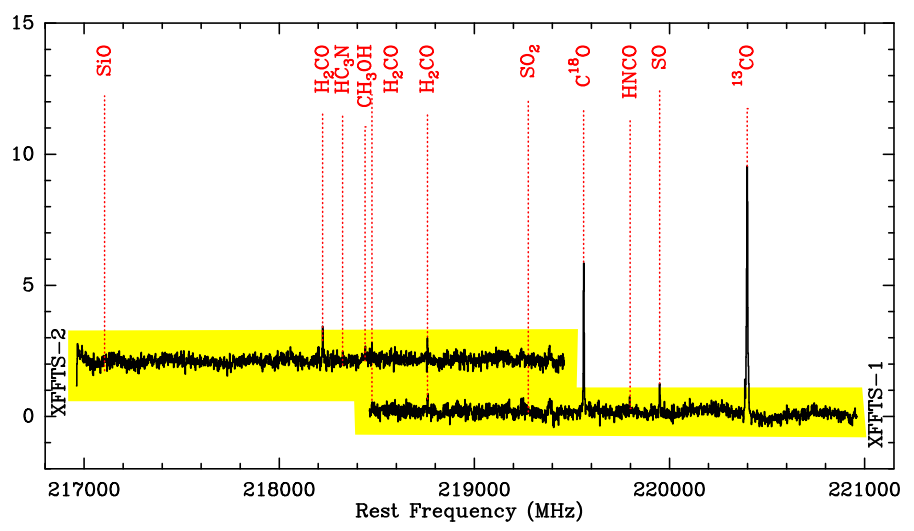

Fig. 2. $4 \mathrm{GHz}$ frequency coverage with the two backend units, XFFTS1 , and -2 . The spectra have been extracted and averaged within the beam towards the brightest ATLASGAL source in the science demonstration field, AGAL340.054-00.244 (Urquhart et al. 2014a, see also Fig. 8). Red labels mark the brightest spectral lines expected in the observed spectral coverage. The spectrum recorded by XFFTS-2 has been offset for clarity.

these filaments, with the gas being guided through magnetic field lines (e.g. Nagai et al. 1998; Nakamura \& Li 2008).

On much larger scales, long molecular filaments stretching up to hundreds of parsecs have been discovered in our Galaxy (Jackson et al. 2010; Goodman et al. 2014; Ragan et al. 2014; Wang et al. 2015; Zucker et al. 2015; Abreu-Vicente et al. 2016). There is no consensus yet on whether these are typically associated with the spiral arms, or instead found in interarm regions. It is also unclear if these two types of filaments (the hundreds of parsec-scale filaments and the small pc-scale filaments associated with star formation), or their formation mechanism are related. Galactic shear probably dominates the shaping of the large-scale filamentary clouds (e.g. Dobbs 2015; Duarte-Cabral \& Dobbs 2016), whereas gravity, turbulence and magnetic fields are more likely to be relevant on the smallest scales; and most likely a mixture of all these processes in between (Federrath 2016).

With a wide coverage of the Galactic plane, and high spatial resolution, the SEDIGISM spectral-line survey will be sensitive to filamentary structures on all scales, down to $\sim 1 \mathrm{pc}$ at the distance to the Galactic centre. Thus, it will be key to providing the much needed kinematical information that will not only allow placing filaments within their Galactic context, but also provide important constraints on the physical properties and initial conditions leading to their formation.

The structure of the paper is as follows: we describe the observations in Sect. 2, and the data reduction pipeline in Sect. 3. We then present some results derived on a science demonstration field, including: extraction and characterisation of molecular clouds (Sect. 4); a study of dense gas clumps (Sect. 5); a preliminary analysis of excitation and physical conditions, based on the combination of the SEDIGISM data with the ThrUMMS survey (Sect. 6); and a study of filamentary structures (Sect. 7). Finally, we summarise our conclsusions and highlight the perspectives of exploiting the full survey data in Sect. 8 .

\section{Observations}

The data has been collected between 2013 and 2015 with the $12 \mathrm{~m}$ diameter Atacama Pathfinder Experiment telescope (APEX, Güsten et al. 2006), located at $5100 \mathrm{~m}$ altitude on Llano de Chajnantor, in Chile. The observations employed the lowest
Table 1. Transitions covered by the instrumental setup.

\begin{tabular}{llcr}
\hline \hline Species & Transition & $\begin{array}{c}\text { Frequency } \\
(\mathrm{GHz})\end{array}$ & $\begin{array}{r}E_{\mathrm{L}} \\
(\mathrm{K})\end{array}$ \\
\hline $\mathrm{CH}_{3} \mathrm{CN}$ & $J=12-11, K=0$ & 220.7473 & 58.3 \\
${ }^{13} \mathrm{CO}$ & $J=2-1$ & 220.3987 & 5.3 \\
$\mathrm{SO}$ & $J=5-4$ & 219.9494 & 24.4 \\
$\mathrm{HNCO}$ & $J=10-9, K=0$ & 219.7983 & 47.5 \\
$\mathrm{C}^{18} \mathrm{O}$ & $J=2-1$ & 219.5604 & 5.3 \\
$\mathrm{SO}_{2}$ & $J=22-23, K=7-6$ & 219.2760 & 342.2 \\
$\mathrm{H}_{2} \mathrm{CO}$ & $\left(3_{2,1}-2_{2,0}\right)$ & 218.7601 & 57.6 \\
$\mathrm{H}_{2} \mathrm{CO}$ & $\left(3_{2,2}-22,1\right)$ & 218.4756 & 57.6 \\
$\mathrm{CH}_{3} \mathrm{OH}$ & $J=4-3, K=2-1 \mathrm{E}$ & 218.4401 & 35.0 \\
$\mathrm{HC}_{3} \mathrm{~N}$ & $J=24-23$ & 218.3247 & 120.5 \\
$\mathrm{H}_{2} \mathrm{CO}$ & $\left(3_{0,3}-2_{0,2}\right)$ & 218.2222 & 10.5 \\
$\mathrm{SiO}$ & $J=5-4$ & 217.1050 & 20.8 \\
\hline
\end{tabular}

Notes. Column 1 lists the molecules; the transitions are described by the main quantum numbers in Col. 2; Cols. 3 and 4 give the rest frequencies and the lower state energies, respectively.

frequency module of the Swedish Heterodyne Facility Instrument (SHFI, Vassilev et al. 2008). This is a single-pixel heterodyne receiver with a sideband-separating mixer operating in a single sideband mode. The back-ends consist of two wideband Fast Fourier Transform Spectrometers (XFFTS; Klein et al. 2012). Each spectrometer covers $2.5 \mathrm{GHz}$ instantaneous bandwidth, with 32768 spectral channels. At the frequency of our observations $(219 \mathrm{GHz})$, this translates to a velocity resolution of $\sim 0.1 \mathrm{~km} \mathrm{~s}^{-1}$. The two spectrometers cover the $4 \mathrm{GHz}$ IF bandwidth of the receiver with an overlap of $500 \mathrm{MHz}$, as shown in Fig. 2. In addition to the CO-isotopologue lines, the setup also covers transitions from several other molecules, including shock enriched molecules $(\mathrm{SiO}, \mathrm{SO})$ and dense gas tracers $\left(\mathrm{H}_{2} \mathrm{CO}\right.$, $\mathrm{CH}_{3} \mathrm{OH}, \mathrm{CH}_{3} \mathrm{CN}$ ) - see Table 1 for rest frequencies and energies. These lines provide diagnostic tools of star formation activity towards the densest regions, tracing for example molecular outflows, shocks, and infall motions.

The survey covers a total of $78 \mathrm{deg}^{2}$ of the southern Galactic plane $\left(-60^{\circ} \leq \ell \leq 18^{\circ},|b| \leq 0.5^{\circ}\right)$, with a $28^{\prime \prime}$ beam. Our observing strategy consisted in dividing the full area to be surveyed into $0.5 \times 0.5 \mathrm{deg}^{2}$ fields. Each field was covered twice with on-the-fly mapping, scanning in two orthogonal directions: along Galactic longitude and latitude. Using a scanning speed of $2^{\prime} / \mathrm{s}$, and $15^{\prime \prime}$ steps between lines (i.e. almost half-beam sampling), the integration time per beam amounts to $0.34 \mathrm{~s}$. With these scanning parameters, we typically reach in the final, combined data a $1-\sigma$ rms noise of $0.8 \mathrm{~K}$ (in main beam brightness temperature scale, $T_{\mathrm{mb}}$; see Güsten et al. 2006) at $0.25 \mathrm{~km} \mathrm{~s}^{-1}$ spectral resolution in average weather conditions, meaning with a total amount of precipitable water vapour (PWV) up to $3 \mathrm{~mm}$.

Using the RADEX code (van der Tak et al. 2007), assuming a kinetic temperature of $20 \mathrm{~K}$, representative of the wide temperature range found in molecular clouds (e.g. Sect. 5.1.2 in Heyer \& Dame 2015), a density of $10^{3} \mathrm{~cm}^{-3}$, and standard abundances $[\mathrm{CO}] /\left[\mathrm{H}_{2}\right]=10^{-4}$ and ${ }^{12} \mathrm{C} /{ }^{13} \mathrm{C}=60$, we estimate that the SEDIGISM survey can make a $3-\sigma$ detection of gas with $\mathrm{H}_{2}$ column densities above $\sim 3 \times 10^{21} \mathrm{~cm}^{-2}$ (or $\sim 60 M_{\odot} \mathrm{pc}^{-2}$ ) in the ${ }^{13} \mathrm{CO}(2-1)$ line, and a 3- $\sigma$ detection of gas above $10^{22} \mathrm{~cm}^{-2}$ (or $\left.\sim 200 M_{\odot} \mathrm{pc}^{-2}\right)$ in $\mathrm{C}^{18} \mathrm{O}(2-1)$. The column density threshold for star formation being of the order of $5 \times 10^{21} \mathrm{~cm}^{-2}$ (e.g. Lada et al. 2010), this sensitivity is well suited to detect all the molecular structures associated with star formation and their surrounding medium. 


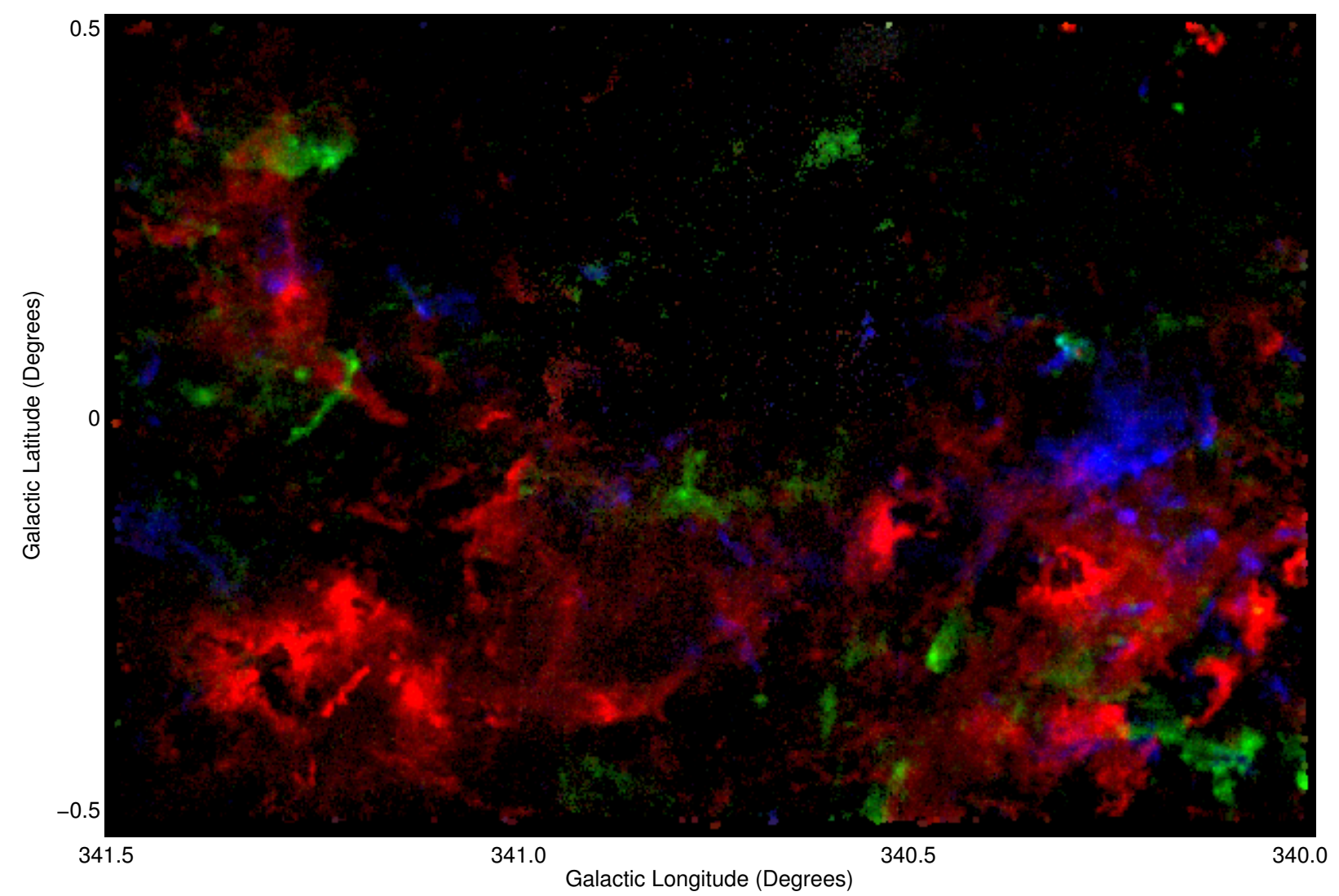

Fig. 3. Three-colour peak ${ }^{13} \mathrm{CO}$ emission map of the science demonstration field. The velocity ranges used to produce the blue, green and red images are -130 to $-110 \mathrm{~km} \mathrm{~s}^{-1},-110$ to $-60 \mathrm{~km} \mathrm{~s}^{-1}$ and -60 to $+5 \mathrm{~km} \mathrm{~s}^{-1}$, respectively. The emission in these velocity ranges is dominated by the near-sides of the $3 \mathrm{kpc}$, Norma and Scutum-Centaurus arms, respectively.

To show a typical example of the data products, and to illustrate the potential of the survey, we selected a $1.5^{\circ} \times 1^{\circ}$ area between $\ell=340.0^{\circ}$ and $\ell=341.5^{\circ}$ (see the line of sight marked in Fig. 1), hereafter referred to as the science demonstration field. This is a representative sample of the full survey: the line of sight in this direction crosses several Galactic arms, so that structures are detected at various distances. The peak of the ${ }^{13} \mathrm{CO}(2-1)$ emission in three representative ranges of $v_{\text {lsr }}$ is shown in Fig. 3. The ${ }^{13} \mathrm{CO}(2-1)$ and $\mathrm{C}^{18} \mathrm{O}(2-1)$ cubes covering the science demonstration field are available for download from a dedicated server hosted by the MPIfR ${ }^{2}$.

The distribution of rms noise values in this region is shown in Fig. 4, and the spatial variations of the rms can be seen in Fig. 5. Most observations of this field were done with PWV < $2 \mathrm{~mm}$, resulting in rms noise of order $1.3 \mathrm{~K}$ in individual scans, and $0.7-0.8 \mathrm{~K}$ in the combined data. However, one sub-field, centred at $(\ell, b)=(340.75,+0.25)$, was observed with $\mathrm{PWV} \approx$ $3.4 \mathrm{~mm}$, which results in a slightly higher noise around $1.7 \mathrm{~K}$ in individual scans, and $\sim 1.2 \mathrm{~K}$ in the combined map.

\section{Data reduction}

The data provided by the APEX telescope consist of spectra calibrated in antenna temperature scale $\left(T_{\mathrm{A}}^{*}\right)$, written in files readable by the CLASS software from the GILDAS package ${ }^{3}$. We created a pipeline in CLASS to process each scan individually, starting from the data expressed in $T_{\mathrm{A}}^{*}$ scale. This pipeline automatically

\footnotetext{
2 http://sedigism.mpifr-bonn.mpg.de/
}

http://www.iram.fr/IRAMFR/GILDAS/

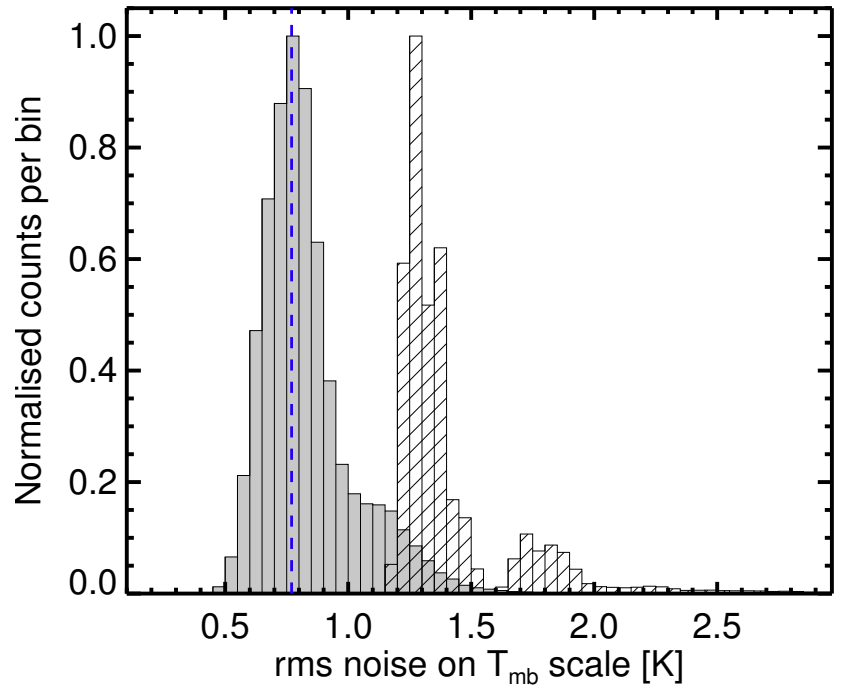

Fig. 4. Distribution of rms noise values for the science demonstration field on $T_{\mathrm{mb}}$ scale. The filled grey histogram shows the noise in the final, combined data; the hatched histogram shows the noise measured in the individual scans that were combined for the final data cube. The distribution peaks at $0.78 \mathrm{~K}$, as indicated by the blue dashed line. Both distributions have been normalised to a peak value of 1 .

detects line emission in order to mask the corresponding channels before baseline subtraction. The main steps of the data reduction are detailed in the next section. 




Fig. 5. Spatial variations of the rms noise in the science demonstration field.

\subsection{Baseline subtraction and calibration}

The processing of each individual scan involves the following steps:

- First, a $\pm 500 \mathrm{~km} \mathrm{~s}^{-1}$ velocity range centred on the ${ }^{13} \mathrm{CO}(2-1)$ line rest frequency is extracted from the spectra; this range is expected to cover all the Galactic emission. Simultaneously, spectra centred on the other spectral lines are extracted from the data, but with a smaller velocity range of $\pm 300 \mathrm{~km} \mathrm{~s}^{-1}$.

- The spectra are re-sampled to $0.25 \mathrm{~km} \mathrm{~s}^{-1}$ velocity resolution for the CO isotopologues, and $0.5 \mathrm{~km} \mathrm{~s}^{-1}$ for the other lines, in order to improve the sensitivity for the weaker lines.

- The calibration of observations done between March and June 2014 have been affected by technical issues with the receiver. Appropriate scaling factors are thus applied to recalibrate the data ${ }^{4}$. These factors are 0.73 and 0.90 for the XFFTS-1 and XFFTS-2 backends, respectively, for data taken between March 20 and April 23, 2014; and 0.80 and 0.95 for data taken between April 23 and June 13, 2014.

- For spectral lines covered by both backends, the data recorded with XFFTS-1 is used, because this backend shows a more stable behaviour compared to XFFTS-2, even in relatively poor weather conditions (i.e. PWV up to $\sim 4 \mathrm{~mm}$ ).

- The rms noise per channel, $\sigma_{\text {rms }}$, is then determined by taking the minimum of the median noise level in a sliding spectral window of 80 channels, expected to be representative of line-free frequency ranges. To increase the signal to noise ratio for the subsequent line finding, this is done on a smoothed version of the data, where the spectra have been spatially averaged in a box of 500" around every given offset.

- The channels that show a signal above a threshold of $3 \times$ $\sigma_{\text {rms }}$ are used to define the windows, which are subsequently excluded for baseline subtraction.

- To increase the efficiency of the data reduction pipeline, the same windows are used for three subsequent pointing offsets, since the data were dumped at every position spaced by $\sim 1 / 3$ of the beam. The same windows are applied to all extracted spectral lines.

- A polynomial baseline of 3rd order is then subtracted from the spectra extracted around all spectral lines.

- Then, the spectra are converted to main beam temperature $\left(T_{\mathrm{mb}}\right)$ scale using an efficiency value of $0.75^{5}$.

\footnotetext{
4 http://www .apex-telescope.org/heterodyne/shfi/ calibration/calfactor/

5 http://www.apex-telescope.org/telescope/efficiency/ index.php
}

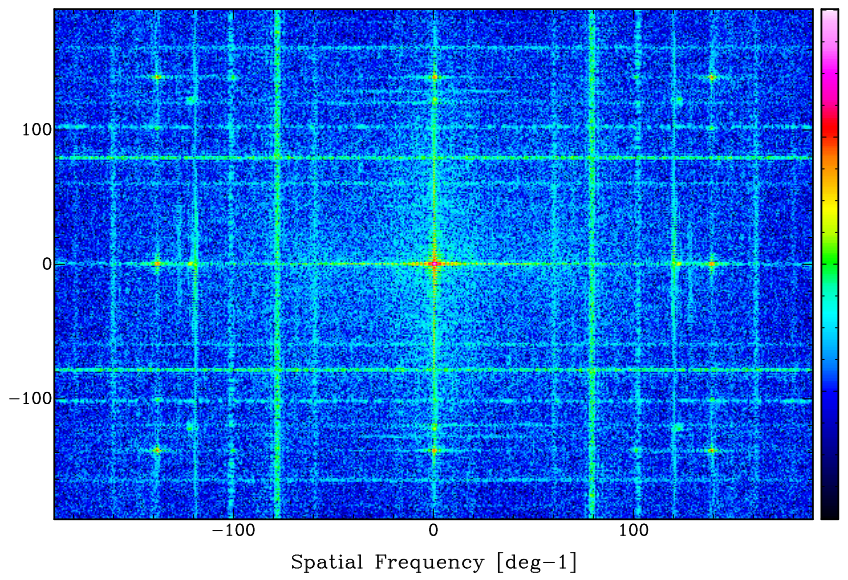

Fig. 6. Two-dimensional power spectrum of the noise image shown in Fig. 5.

- Finally, emission from the reference position is corrected for, when necessary (see below).

The observations for the SEDIGISM survey used absolute reference positions, located at $\pm 1.5^{\circ}$ in $b$, at the same $\ell$ as the centre of each field. These reference positions are usually far enough from the Galactic plane to be located towards emission-free regions of the sky, but not all reference positions are clear of emission in the ${ }^{13} \mathrm{CO}(2-1)$ line. Therefore, all the reference positions have been observed and reduced independently, using a more distant reference position off the Galactic plane. Only towards those fields where the reference position shows emission in ${ }^{13} \mathrm{CO}(2-1)$, this emission has been corrected for in the final maps. We independently checked for emission in the $\mathrm{C}^{18} \mathrm{O}(2-1)$ line toward the reference positions as well, and corrected for it when necessary. Still, we cannot exclude that negative artefacts due to imperfect correction for emission at the reference position, may still be present in some data.

Two line-rich sources (Sgr B2(N) and IRC+10216) were regularly observed in 2014 and 2015, using the same set-up as for the science observations. These sources can be used as spectral calibrators. We measure a dispersion of $\sim 7 \%$ in the integrated area below the ${ }^{13} \mathrm{CO}(2-1)$ line between observations, for both sources. Therefore, we can safely assume that the uncertainty on the temperature scale is better than $10 \%$, as is typically the case with the SHFI instrument.

\subsection{Combining several scans}

Two scans observed with orthogonal scanning directions cover each given field. When the noise obtained by combining these two scans was significantly higher than average, we observed the field again at least in one scanning direction. The reduced, calibrated data obtained from the different scans are finally combined and gridded using 9.5" cell size. The gridding process includes a convolution with a Gaussian kernel of size one-third the telescope beam. This provides data cubes with a final angular resolution of $30^{\prime \prime}$. Data cubes are generated for all the transitions listed in Table 1 , at $0.25 \mathrm{~km} \mathrm{~s}^{-1}$ resolution for the CO isotopologues, and $0.5 \mathrm{~km} \mathrm{~s}^{-1}$ resolution for the other species.

Combining several scans observed with different scanning directions helps in reducing striping artefacts in the data. However, the pixel-to-pixel noise is not independent but shows high degree of correlation at some specific spatial scales. This is illustrated in Fig. 6, which shows the 2D power spectrum (the square 


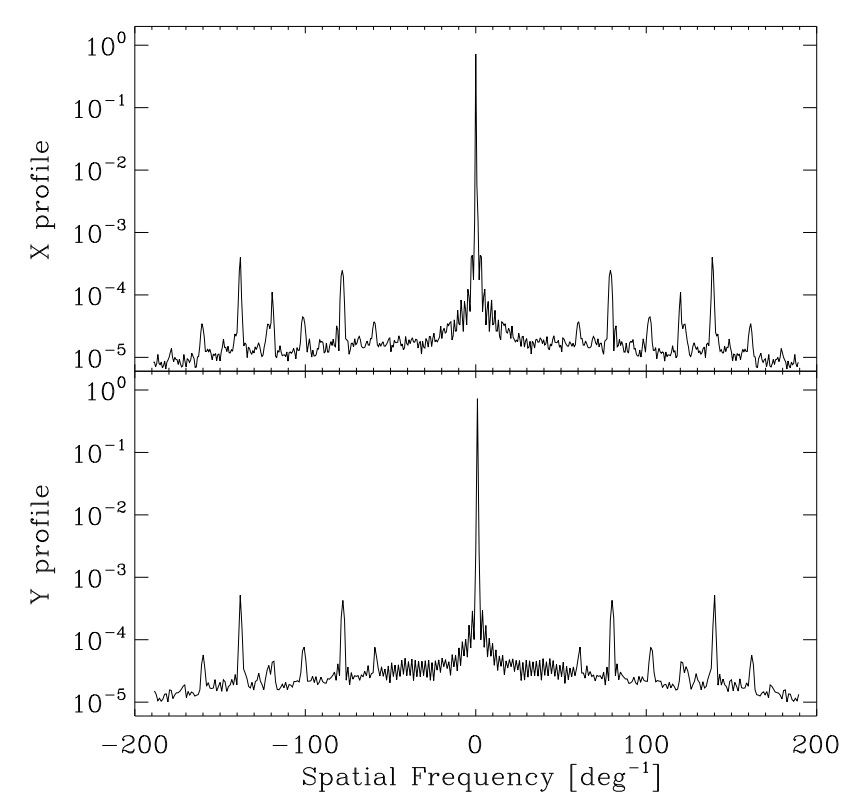

Fig. 7. Power spectrum of the noise image, as shown in Fig. 6, but averaged over Galactic latitudes (top) and longitudes (bottom).

of the module of the 2D Fourier transform) of the noise image shown in Fig. 5. Some peaks are clearly visible (see also Fig. 7) at frequencies corresponding to multiples of the scanning parameters (steps between lines, and steps between dumps within a line).

\subsection{Lines from other species}

The velocity ranges where a signal was automatically detected in the ${ }^{13} \mathrm{CO}(2-1)$ data have been masked for subtracting baselines to the data covering all the other lines possibly present in the data. Some lines from other species are indeed detected towards the brightest clumps, including $\mathrm{SO}(5-4), \mathrm{H}_{2} \mathrm{CO}$ (three different lines), and one line of $\mathrm{CH}_{3} \mathrm{OH}$. In the science demonstration field, these are seen only toward a handful of sources; an example is shown in Fig. 8. Due to low detection statistics, these lines will not be further discussed in this overview paper.

\section{Molecular clouds and complexes}

\subsection{The extraction algorithm: SCIMES}

To extract clouds from the SEDIGISM science demonstration field, we have used the SCIMES algorithm (Spectral Clustering for Interstellar Molecular Emission Segmentation; for details see Colombo et al. 2015). This tool is designed to identify molecular cloud complexes in 3D data cubes, based on cluster analysis. While other available 3D cloud extraction algorithms tend to segment the emission into individual emission peaks/clumps inside molecular clouds, the advantage of SCIMES is that it is tailored to group different peaks together, making it more suitable to extract large complexes of clouds.

In practice, this code considers the dendrogram tree of the 3D structures in the data cube (as per the implementation of Rosolowsky et al. 2008, to analyse astronomical data sets) in the broader framework of graph theory, and groups different leaves (in this case, the emission peaks) together into "clusters" of leaves, based on some criteria (e.g. intensity, luminosity, or volume). For a more detailed description of the terminology and the algorithm, see Colombo et al. (2015).

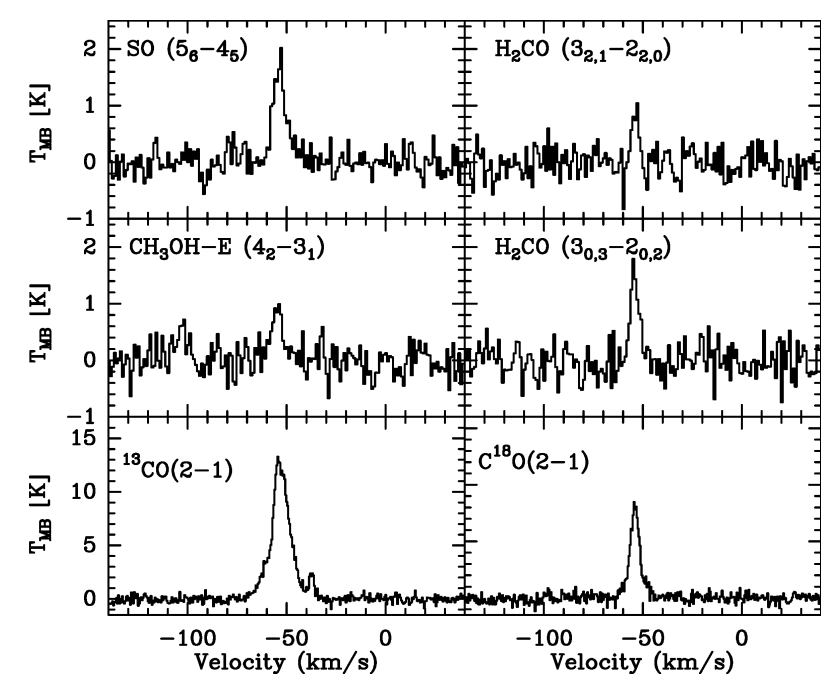

Fig. 8. Spectra extracted around the lines of $\mathrm{SO}, \mathrm{H}_{2} \mathrm{CO}, \mathrm{CH}_{3} \mathrm{OH}$, ${ }^{13} \mathrm{CO}$ and $\mathrm{C}^{18} \mathrm{O}$ towards AGAL340.054-00.244, which is the brightest ATLASGAL clump in the field. The emission has been integrated within a radius of $15^{\prime \prime}$ around the peak position of the dust clump.

We ran the extraction of clouds on the ${ }^{13} \mathrm{CO}(2-1)$ emission $\ell b v$ data cube of the SEDIGISM science demonstration field, after binning the velocities into $0.5 \mathrm{~km} \mathrm{~s}^{-1}$ channels, resulting in a data cube with a noise level ranging between $\sim 0.6-0.8 \mathrm{~K}$. For building the dendrogram tree of the data cube, we have therefore considered an rms noise level $(\sigma)$ of $0.7 \mathrm{~K}$. We have used a value of 4- $\sigma$ as the minimum difference between two peaks for these to be considered as separate leaves, and a lower threshold for detection of $2-\sigma$, to maximise the connections between different structures at contiguous lower intensity levels. We used both the intensity and volume as the SCIMES clustering criteria. This extraction provides an output data cube with a mask containing all the SCIMES clusters found, a data cube with the mask of only the dendrogram leaves, and the entire catalogue of dendrogram structures. As we were also interested in the larger clouds that may simply have little substructure within them (the level of sub-structuring can be a consequence of the resolution), we have also included the single leaves in the dendrogram which had been excluded by the clustering algorithm, but whose size was large enough to be well resolved (i.e. when 1- $\sigma$ of the semimajor axis of the cloud was larger than the beam size).

\subsection{Catalogue of molecular clouds and measured properties}

We extracted a total of 182 molecular clouds, of which 58 are categorised as clusters by SCIMES (i.e. complexes with at least 2 dendrogram leaves clustered together), and 124 are wellresolved single-leaf clouds. The position and extent of these clouds can be seen as coloured masks in the $\ell b$ and $\ell v$ plots of Fig. 9, where the left panels show the SCIMES molecular cloud complexes alone, while the right panels also include the well resolved single-leaf clouds.

We measured the following properties for each cloud: centroid position in Galactic coordinates, velocity, velocity dispersion, intensity-weighted semi-major and semi-minor axes, and respective position angle. The aspect ratio is then computed as the ratio of the major/minor axis. These properties are summarised in Table 2 for a sub-sample of the largest GMCs (full catalogue in Table A.1). We also estimate the physical properties 

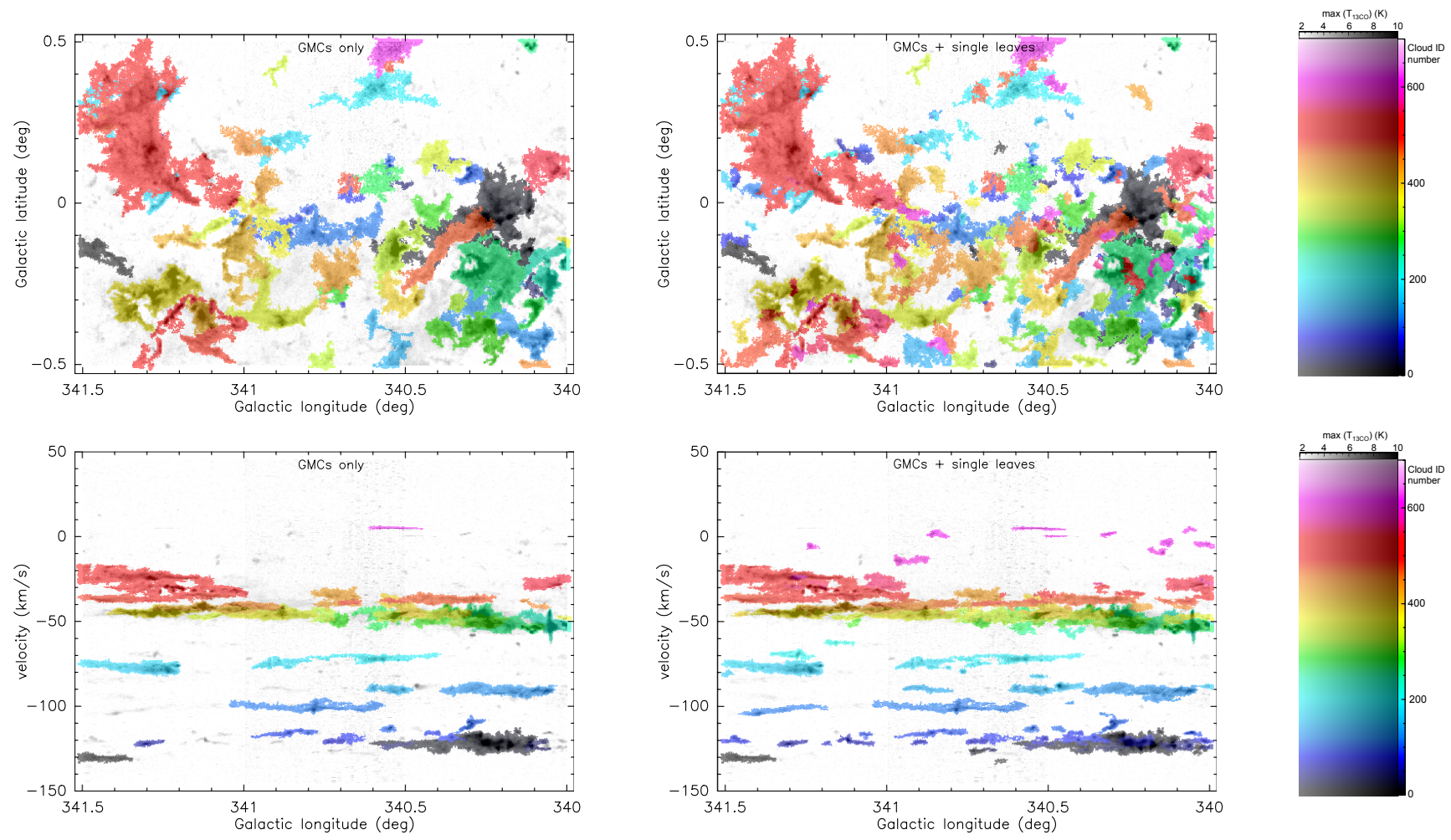

Fig. 9. Position and extent of the SCIMES cloud complexes only (left), and all the clouds (right), i.e. the molecular cloud complexes plus all the well resolved single-leaf clouds, in $\ell b$ (top) and $\ell v$ space (bottom). The colour-coding refers to the ID number of the clouds as per our catalogue, and relates to the third dimension of the cube (i.e. $v_{\mathrm{lsr}}$ ). The coloured masks of the clouds are overplotted with transparency over the maps of the peak ${ }^{13} \mathrm{CO}(2-1)$ emission, in grey scale (see the transparency-combined scale on the right).

of our sample of clouds (see Sect. 4.4), after determining their kinematic distances (Sect. 4.3).

In order to validate the cloud catalogue built using the SCIMES algorithm, we compared the results of the SCIMES extraction with independently, visually identified structures in the ${ }^{13} \mathrm{CO}(2-1) \ell b v$ cubes. To do this, we started by identifying the brightest peaks of emission in the $\ell b v$ cube and determined their extent in velocity. We then defined polygons around individual structures based on their morphology and calculated their corresponding average spectra, to which we fit single or multiple Gaussian profiles. For the final identification we mapped the structures by integrating the emission over the FWHM of the fitted lines, and considering the emission above a threshold of $\sim 3-\sigma$ noise level. With this method, we identified a total of 25 bright structures.

By comparing the position and extent of these structures in $\ell b v$ to the SCIMES clouds, we find that ten of them have a one to one correspondence, while in five cases, one visually identified structure corresponds to two SCIMES clouds. The remaining structures cover similar emission to the SCIMES clouds, but the dissection in position and velocity differs slightly, mostly with the SCIMES algorithm tending to segment the emission more in position space, and group it more efficiently in the velocity space. Nevertheless, we find that in general the SCIMES extraction provides a satisfactory segmentation of the data.

\subsection{Distance determination}

We have derived the kinematic distances of all the molecular clouds in the science demonstration field using the Galactic rotation model of Brand \& Blitz (1993). For sources located within the Solar Circle, there are two possible solutions equally spaced on either side of the tangent distance; these are known as the near and far distances. To resolve these kinematic distance ambiguities, we have used the HI self-absorption method (HISA; e.g. Jackson et al. 2002; Roman-Duval et al. 2009; Wienen et al. 2015). This works on the premise that the cold HI associated with a source at the near distance will produce a dip in the warmer HI emission, which arises from warm gas located throughout the Galactic mid-plane, at the same velocity as the source (see Fig. 10 for example profiles).

For this purpose, we made use of HI maps from the Southern Galactic Plane Survey (SGPS; McClure-Griffiths et al. 2005) to determine the existence of HISA towards all clouds, directly from the 3-dimensional data cube. If a cloud showed a strong HI absorption dip within the cloud mask when compared to the immediate surroundings (from the HI cube), the cloud was considered to have strong HISA, and was placed at the near distance. Otherwise, the HISA criterion was deemed ambiguous, in which case we made use of the distance tool being developed by the Hi-GAL/VIALACTEA project. The VIALACTEA automatic distance tool is still in development but will be publicly available in the course of 2017 (Russeil et al., in prep.). This tool combines pre-existing distance information gathered from catalogues found in the literature (e.g. maser parallax distance, spectrophotometric distance of HII regions, HISA solution for the kinematic distance ambiguity, IRDC associations, etc.) and new 3D extinction data cubes produced following Marshall et al. (2006). If the distances provided by the Hi-GAL distance tool were robust for clouds with ambiguous HISA (e.g. due to the match with an IRDC, which are considered to be predominately located in the foreground with respect to the Galactic Centre), the distance was taken as the Hi-GAL distance tool solution. If the distance ambiguity was not solved with either Hi-GAL 
Table 2. Properties of a sub-sample of clouds from the SCIMES extraction, restricted to GMCs with at least 5 leaves.

\begin{tabular}{|c|c|c|c|c|c|c|c|c|c|c|c|c|c|c|c|c|}
\hline \multirow[b]{2}{*}{ ID } & \multirow[b]{2}{*}{ Name } & \multicolumn{8}{|c|}{ Measured properties } & \multicolumn{7}{|c|}{ Physical properties } \\
\hline & & $\begin{array}{c}a \\
\left({ }^{\prime \prime}\right)\end{array}$ & $\begin{array}{c}b \\
\left({ }^{\prime \prime}\right)\end{array}$ & $\begin{array}{l}\text { PA } \\
\left({ }^{\circ}\right)\end{array}$ & AR & $\begin{array}{c}v_{\mathrm{lsr}} \\
\left(\mathrm{km} \mathrm{s}^{-1}\right)\end{array}$ & $\begin{array}{c}\sigma_{v} \\
\left(\mathrm{~km} \mathrm{~s}^{-1}\right)\end{array}$ & $\begin{array}{c}<W_{\mathrm{CO}}> \\
\left(\mathrm{K} \mathrm{km} \mathrm{s}^{-1}\right)\end{array}$ & $N_{\mathrm{l}}$ & $\begin{array}{c}d \\
(\mathrm{kpc})\end{array}$ & $\begin{array}{l}\text { Area } \\
\left(\mathrm{pc}^{2}\right)\end{array}$ & $\begin{array}{c}R \\
(\mathrm{pc})\end{array}$ & $\begin{array}{l}l_{\max } \\
(\mathrm{pc})\end{array}$ & $\begin{array}{c}M \\
\left(10^{3} M_{\odot}\right)\end{array}$ & $\begin{array}{c}\Sigma \\
\left(M_{\odot} \mathrm{pc}^{-2}\right)\end{array}$ & $\alpha_{\mathrm{vir}}$ \\
\hline 15 & SDG340.245-0.056 & 322 & 203 & -161 & 1.6 & -122.0 & 2.3 & 9.7 & 14 & $6.58 \pm 0.28$ & 915 & 17.1 & 72.2 & 196.9 & 214 & 0.5 \\
\hline 34 & SDG & 76 & 69 & 110 & 1.1 & -123.2 & 1.8 & 4.0 & 5 & $6.63 \pm 0.29$ & 51 & 4.1 & 14.5 & 4.7 & 89 & 3.3 \\
\hline 127 & SDG & 364 & 146 & 153 & 2.5 & -90.7 & 1.6 & 5.1 & 9 & $5.47 \pm 0.26$ & 275 & 9.4 & 44.0 & 1.3 & 113 & 0.9 \\
\hline 234 & SDG & 151 & 105 & 70 & 1.4 & -51.9 & 2.6 & 15.8 & 5 & $3.86 \pm 0.36$ & 62 & 4.5 & 17.6 & 22.0 & 350 & 1.6 \\
\hline 260 & SDG & 180 & 147 & 148 & 1.2 & -48.9 & 3.5 & 17.4 & 9 & $3.73 \pm 0.37$ & 137 & 6.6 & 23.8 & 53.3 & 386 & 1.7 \\
\hline 271 & $2+0.069$ & 114 & 88 & -157 & 1.3 & -47.1 & 3.6 & 3.3 & 6 & $3.66 \pm 0.38$ & 38 & 3.5 & 13.5 & 2.8 & 73 & 18.4 \\
\hline 298 & SDG340.300-0.395 & 228 & 92 & 176 & 2.5 & -49.1 & 2.9 & 14.3 & 7 & $3.74 \pm 0.37$ & 76 & 4.9 & 20.1 & 24.1 & 317 & 2.0 \\
\hline 375 & SDG341.260-0.276 & 238 & 107 & -153 & 2.2 & -44.4 & 1.2 & 12.5 & 8 & $3.57 \pm 0.40$ & 105 & 5.8 & 23.0 & 29.2 & 277 & 0.3 \\
\hline 408 & SDG341.010-0.151 & 229 & 110 & 89 & 2.1 & -42.3 & 1.1 & 6.1 & 6 & $3.44 \pm 0.41$ & 70 & 4.7 & 20.2 & 9.5 & 134 & 0.7 \\
\hline 508 & SDG341.212-0.345 & 146 & 42 & -136 & 3.4 & -30.4 & 1.6 & 5.5 & 5 & $2.00 \pm 0.49$ & 7 & 1.5 & 7.7 & 0.9 & 121 & 5.2 \\
\hline 525 & SDG341.327+0.219 & 422 & 219 & 104 & 1.9 & -23.6 & 2.2 & 6.2 & 13 & $2.27 \pm 0.56$ & 147 & 6.9 & 26.7 & 20.4 & 138 & 1.9 \\
\hline
\end{tabular}

Notes. The ID number shows the catalogue number associated with the cloud (same as the colour-scale in Fig. 9). The GMC name is defined as SDG (for SEDIGISM) followed by the Galactic coordinates of the clouds' centroid. Columns 3 and 4 show the intensity-weighted semi-major and semi-minor axes, $a$ and $b$, respectively; Column 5 shows the position angle (PA), and Col. 6 shows the aspect ratio (AR) defined as $a / b$. In Cols. 7-9 we show the centroid velocity, velocity dispersion, and average ${ }^{13} \mathrm{CO}(2-1)$ integrated intensity across the area of the cloud. Column 10 shows the number of dendrogram leaves, $N_{\mathrm{l}}$, that make up each GMC. Column 11 shows the adopted distances $(d)$ and their uncertainties. Columns 12-14 show the exact area defined by the clouds' masks, the equivalent radius $(R$, assuming circular geometry) and maximum length $\left(l_{\max }\right)$. Columns 15-17 show the total mass $(M)$, the average surface density $(\Sigma)$, and the virial parameter $\left(\alpha_{\mathrm{vir}}\right)$. See Sect. 4.4 for details.
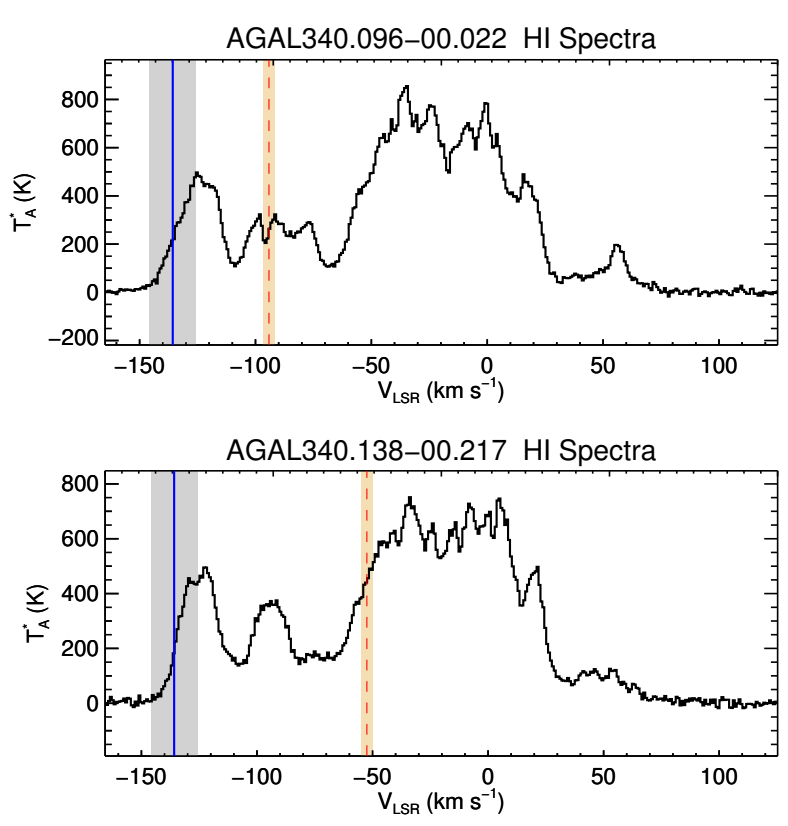

Fig. 10. Example HI spectra extracted towards dense clumps identified from the ATLASGAL survey. The velocity of the tangent position is determined from a fit to HI data (e.g. McClure-Griffiths \& Dickey 2007) and is indicated by the blue line; the grey shaded region covers a velocity range of $\pm 10 \mathrm{~km} \mathrm{~s}^{-1}$. Sources in this region are placed at the distance of the tangent point. The dashed red vertical line shows the velocity of the source with the yellow shaded region showing the typical FWHM of the molecular lines. The top and bottom panels show examples of a source located at the near and far distances, respectively.

or HISA, but there was a match with an ATLASGAL source for which the distance ambiguity had been solved independently (see Sect. 5.1), we took the ATLASGAL distance. In cases where none of these three methods could solve the distance ambiguity, we chose the far distance as the final distance of the cloud, because clouds located at the far distance are less likely to give rise to observable signatures (e.g. HISA or IRDC).
The distances that we have determined for each cloud are listed in Table A.1. Ten clouds in our sample are located on the Solar circle (i.e. $\left|v_{\mathrm{lsr}}\right|<10 \mathrm{~km} \mathrm{~s}^{-1}$ ), so that no reliable kinematic distance can be determined; these have been excluded from our analysis. For the remaining clouds, the quoted uncertainty simply comes from the rotation model and an assumed uncertainty of $7 \mathrm{~km} \mathrm{~s}^{-1}$ on the velocity, representative of typical departure from circular motions induced by spiral density waves or local peculiar motions. However, there is also an intrinsic uncertainty associated with the method of using velocities as proxy for the distance, as well as uncertainties that depend on the origin of the distance determination (e.g. maser or stellar parallax distances, or simply an HiSA/extinction distance). For simplicity, we assume that the overall distance uncertainty is of the order of $30 \%$.

From the 35 clouds that contain at least one ATLASGAL clump, 24 clouds have distances that agree with all three methods (HISA method, Hi-GAL tool and ATLASGAL solution), ten clouds have one method disagreeing (five Hi-GAL far distances were revised to a near distance, and five far ATLASGAL distances were brought to the near distances), and one cloud with a far distance assigned by both Hi-GAL and ATLASGAL was brought to the near distance by our HISA method. For the remaining 147 clouds in our catalogue, 131 have distances that agree between the HISA method and the Hi-GAL distance tool determinations (although this number includes 26 clouds for which neither method had solved the distance ambiguity), and 16 distances have been revised from the Hi-GAL far distances to a near distance with the presence of strong HISA. A tag indicating how the distance ambiguity was solved is included in the final catalogue (Table A.1).

\subsection{Physical properties of the clouds}

Having determined the distances to all the clouds, we estimate their physical properties, which we list in Table 2 for our subsample (full catalogue in Table A.1). In particular, Table 2 lists the exact area as covered by the mask of each cloud, the equivalent radius (if taking a circular geometry), and the projected length of the cloud, measured between the two most distant points within the cloud. 

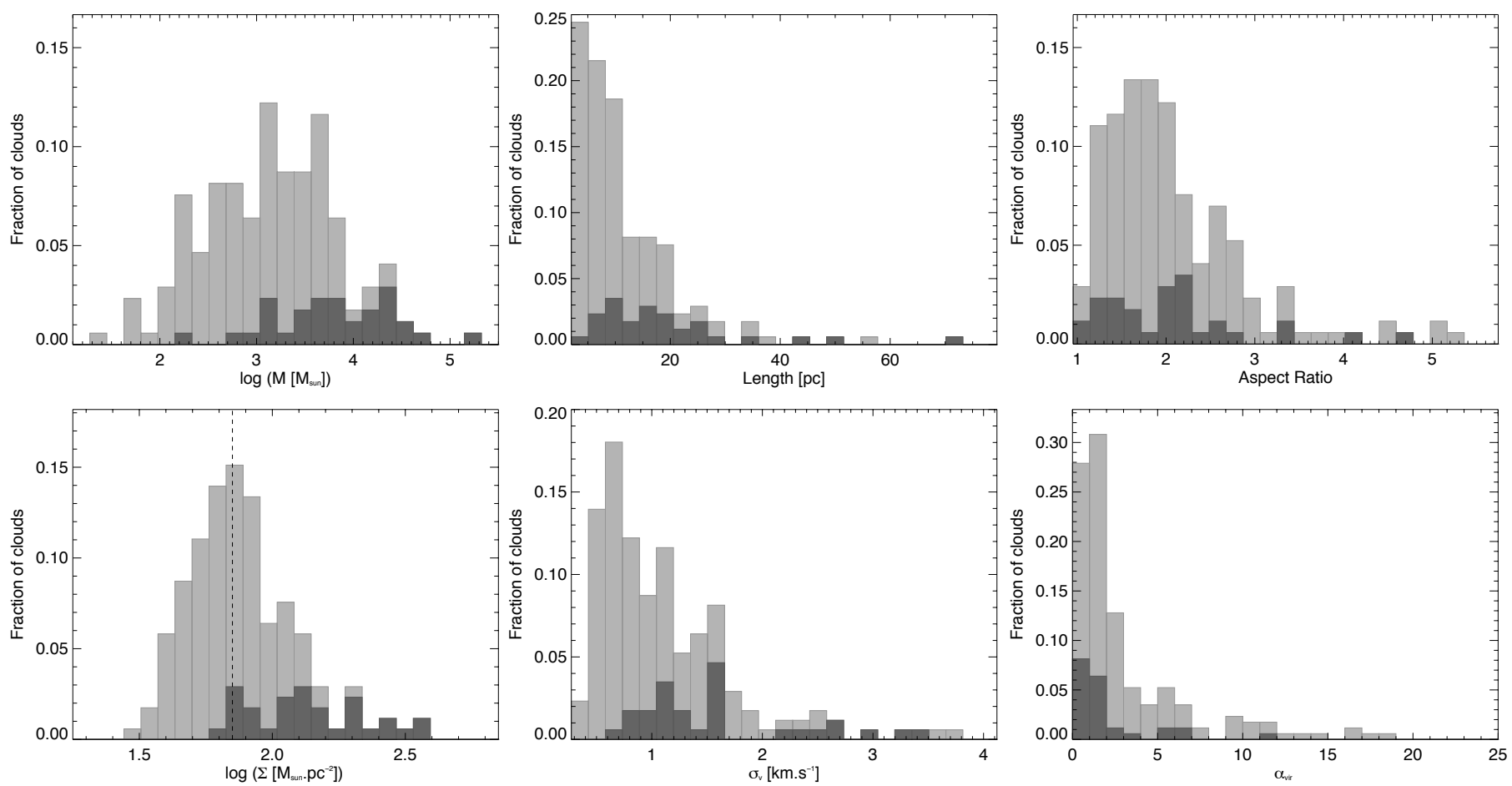

Fig. 11. Properties of the GMCs within the science demonstration field: distributions of masses $M$ (top-left), maximal lengths (top-centre), aspect ratios (top-right), average mass surface densities $\Sigma$ (bottom-left), velocity dispersions $\sigma_{v}$ (bottom-centre), and virial parameters $\alpha_{\text {vir }}$ (bottom-right). The light-grey histograms show the distributions of properties for all clouds, excluding ten clouds with $\left|v_{\mathrm{lsr}}\right|<10 \mathrm{~km} \mathrm{~s}^{-1}$, as those have large distance uncertainties. The dark-grey histograms show the sub-sample of clouds that have an ATLASGAL match. The dashed vertical line on the average surface density plot shows our estimated completeness limit, $\sim 70 \mathrm{M}_{\odot} \mathrm{pc}^{-2}$, corresponding to $\sim 3 \times 10^{21} \mathrm{~cm}^{-2}$.

To estimate the cloud masses, we have converted the integrated intensities of ${ }^{13} \mathrm{CO}(2-1)$ into $\mathrm{H}_{2}$ column densities using an $X_{{ }^{13} \mathrm{CO}(2-1)}$ conversion factor, which we have determined using an ancillary $\mathrm{H}_{2}$ column density map derived from the Hi-GAL survey data (Molinari et al. 2010). This map was built by fitting a pixel-by-pixel grey body curve to the spectral energy distribution from 160 to $500 \mu \mathrm{m}$ (Elia et al. 2013), assuming an opacity law with a fixed spectral index $\beta=2$, and $\kappa_{0}=0.1 \mathrm{~cm}^{2} \mathrm{~g}^{-1}$ at $v_{0}=1200 \mathrm{GHz}$ (Hildebrand 1983). We then estimated the $X_{{ }^{13} \mathrm{CO}(2-1)}$ factor for regions where there was an extracted cloud, and obtained $X_{{ }^{13} \mathrm{CO}(2-1)} \approx 1_{-0.5}^{+1} \times 10^{21} \mathrm{~cm}^{-2}\left(\mathrm{~K} \mathrm{~km} \mathrm{~s}^{-1}\right)^{-1}$. We note that this factor is in remarkable agreement with the one derived by solving the radiative transfer equations for the $J=2-1$ and $J=1-0$ lines of ${ }^{13} \mathrm{CO}$ simultaneously (see Sect. 6.2). Interestingly, this is only a factor of 5 higher than the recommended value for the classical $X_{\mathrm{CO}}$ (Bolatto et al. 2013), although the ${ }^{12} \mathrm{C} /{ }^{13} \mathrm{C}$ isotopic ratio is typically of order 60 ; this is a direct consequence of the large difference in line opacity between ${ }^{12} \mathrm{CO}$ and ${ }^{13} \mathrm{CO}$.

With this $X_{{ }^{13}} \mathrm{CO}(2-1)$, and assuming a molecular weight $\mu_{m \mathrm{H}}$ of 2.8 , we derived the cloud masses, the average gas surface density, $\Sigma$, across each cloud's area, and the virial parameter, defined as (Bertoldi \& McKee 1992):

$\alpha_{\mathrm{vir}}=5 \sigma_{v}^{2} R / G M$,

where $G$ is the gravitational constant, $\sigma_{v}$ the measured velocity dispersion, and $R$ is the equivalent radius. Given the uncertainties on the distance estimates and on the $X_{\mathrm{CO}}$ factor, all these quantities have an uncertainty of at least a factor two.

The distributions of these physical properties for our cloud sample are shown in Fig. 11, and their statistics are summarised in Table 3. From these, we can see that the mass distribution of
Table 3. Summary of the statistical properties of clouds within the science demonstration field.

\begin{tabular}{lccc}
\hline \hline Property & Mean & $\sigma$ & Median \\
\hline $\log \left[M\left(M_{\odot}\right)\right]$ & 3.14 & 0.70 & $3.18 \pm 0.49$ \\
Length $(\mathrm{pc})$ & 11.8 & 10.1 & $9.1 \pm 4.9$ \\
Aspect ratio & 2.1 & 0.80 & $1.87 \pm 0.48$ \\
$\sigma_{v}\left(\mathrm{~km} \mathrm{~s}^{-1}\right)$ & 1.12 & 0.64 & $0.97 \pm 0.37$ \\
$\log \left[\Sigma\left(M_{\odot} \mathrm{pc}^{-2}\right)\right]$ & 1.89 & 0.20 & $1.87 \pm 0.12$ \\
$\alpha_{\text {vir }}$ & 3.1 & 3.6 & $1.8 \pm 1.4$ \\
\hline
\end{tabular}

Notes. Columns 2 and 3 show the mean value of the property listed in Col. 1 and the associated standard deviation. Column 4 shows the median value and the respective mean absolute deviation of the first and third quartiles.

the sample is relatively flat, with masses spanning over four orders of magnitude, from a few tens up to $10^{5} M_{\odot}$. The other distributions are more strongly peaked, with aspect ratios $\$ 2$, and velocity dispersions around $1 \mathrm{~km} \mathrm{~s}^{-1}$. The majority of the clouds have typical lengths of 10-20 pc, although the low-end distribution of lengths is limited by the resolution of the data. Roughly $\sim 60 \%$ of the clouds have $\alpha_{\text {vir }}<2$, half of which with $\alpha_{\text {vir }}<1$. Only a small fraction of clouds $(\sim 20 \%)$ have $\alpha_{\text {vir }}>5$. Considering a factor of two uncertainty on the mass estimates, most of the clouds that we extract may well be self-gravitating structures.

This can be better seen in Fig. 12, where we show the Heyer et al. (2009) correlation between gas surface densities $\Sigma$ and the size-linewidth coefficient (represented here as $\sigma_{v}^{2} / R$ ) for a compilation of Galactic and extragalactic GMCs, as well as the clouds presented here. On the top-left side of this plot, at low surface densities, clouds have large virial parameters, and they are in a so-called pressure-confined regime. At higher gas surface 


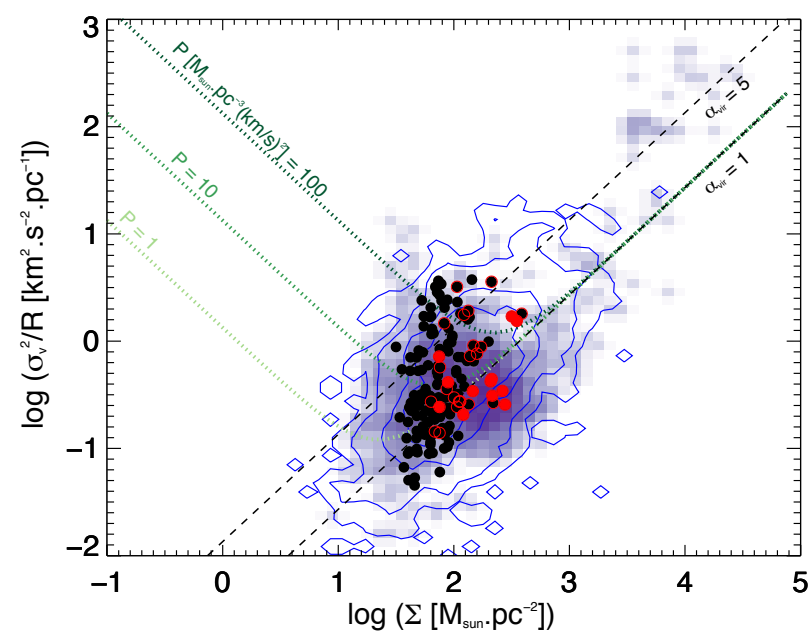

Fig. 12. Characteristic size-linewidth coefficient $\left(\sigma_{v}^{2} / R\right)$ as a function of average gas surface density $\Sigma$ for all resolved GMCs extracted from the science demonstration field (black circles; those outlined in red highlight the clouds with an ATLASGAL counterpart and the filled red circles correspond to clouds showing signs of highmass star formation - see Sect. 5.3 for details). For comparison, the density of points in this plot from a compilation of extragalactic cloud samples (from Rosolowsky et al. 2003; Rosolowsky 2007; Bolatto et al. 2008; Santangelo et al. 2010; Wong et al. 2011; Wei et al 2012; Colombo et al. 2014) and a compilation of Galactic cloud samples (from Heyer et al. 2009; Rathborne et al. 2009; Wang et al 2009; Ginsburg et al. 2012; Giannetti et al. 2013; Battersby et al. 2014; Walker et al. 2015) are shown with blue contours, and with blue colour shading, respectively. The green dotted curves show the expected force balance between kinetic, gravitational and external pressure, for different values of external pressure, from $P=1$ to $100 M_{\odot} \mathrm{pc}^{-3} \mathrm{~km}^{2} \mathrm{~s}^{-2}$ (which corresponds to $P / k \sim 5 \times 10^{3}-5 \times 10^{5} \mathrm{~K} \mathrm{~cm}^{-3}$ ). The black-dashed lines correspond to $\alpha_{\mathrm{vir}}=1$ and $\alpha_{\mathrm{vir}}=5$.

densities, the gravity becomes dominant and clouds cross over to a self-gravitating regime, with lower values of the virial parameter. We can see that our sample of clouds from the SEDIGISM science demonstration field lies in a similar regime as other Galactic and extragalactic clouds, mostly scattered around the $\alpha_{\text {vir }}=1$ line.

One should be aware, however, that the molecular clouds identified from the ${ }^{13} \mathrm{CO}(2-1)$ emission are likely tracing the high-density regions of larger molecular cloud complexes (e.g. Langer et al. 2014; Duarte-Cabral \& Dobbs 2016). In fact, within the science demonstration field, approximately $33 \%$ of the total ${ }^{13} \mathrm{CO}(2-1)$ emission above $3 \sigma$ was not assigned to any particular cloud, and is mostly part of a smoother, more diffuse background connecting several clouds. The extent of this background may be much larger than what we can detect here, not only because some of the underlying molecular gas may be in fact CO-dark, but also because ${ }^{13} \mathrm{CO}(2-1)$ cannot trace the very low column densities.

To address this, we have investigated the emission towards the SEDIGISM science demonstration field with lower excitation energy transitions ( $J=1-0)$, and also higher abundance species $\left({ }^{12} \mathrm{CO}\right)$, to be more sensitive to the lower-columndensity envelope of the clouds. Using the ${ }^{12} \mathrm{CO}(1-0)$ data of the Dame et al. (2001) survey, García et al. (2014) found a total of only 11 GMCs within the science demonstration field, which comprise 172 of the SCIMES clouds. However, the spatial resolution of the Dame et al. (2001) survey is much coarser than that of the SEDIGISM survey (530" compared to 30"). Therefore, some of the GMCs identified by García et al. (2014) could be simply the result of the blending of several clouds that may not be physically connected.

We have also performed a comparison of our cloud catalogue with the clouds detected by the ThrUMMS survey (Barnes et al. 2015 ) in the science demonstration field (with a much better resolution relative to the Dame survey although still $\sim 2.5$ times lower than SEDIGISM), using the same cloud extraction algorithm as used here (SCIMES). We have done so using both the ${ }^{12} \mathrm{CO}(1-0)$ and ${ }^{13} \mathrm{CO}(1-0)$ data and found that the clouds from García et al. (2014) are now sub-divided into smaller clouds, but they still tend to group several SEDIGISM clouds together. The grouping of numerous SEDIGISM clouds into larger GMCs as seen with ${ }^{12} \mathrm{CO}$ or lower transitions of ${ }^{13} \mathrm{CO}$ seems to confirm the existence of a large scale diffuse molecular gas, which connects the different peaks extracted from SEDIGISM.

\subsection{Galactic distribution of molecular clouds}

The positions of all the molecular clouds extracted from the science demonstration field are shown in Fig. 13, overlaid on a customised model of the spiral arms. If we were to take a typical 4-armed symmetric logarithmic spiral potential with a pitch angle of 12.5 degrees, as found to be the best fit of the emission of the entire Galaxy by Pettitt et al. (2014), based on fitting hydrodynamical models to the low-resolution maps from Dame et al. (2001), the emission in the science demonstration field would not be fitted particularly well, especially for the near Norma and near Scutum-Centaurus arms. There are a number of possible reasons for this: $i$ ) a global fit of log-normal spirals is unlikely to fit small fields accurately; $i$ ) the spiral arms shown are based on the stellar potential, and gas response is much more complicated than simply tracing the bottom of the potential well; and iii) these spiral arms are based on a simple circular rotation model, whereas observations (and numerical simulations) suggest a more complex velocity field with many smaller scale undulations and non-circular velocities.

To improve the agreement with the data, we have altered the symmetric 4-armed model by slightly rotating (by 15 degrees) the azimuthal locations of the Norma and Scutum-Centaurus arms; the resulting model is shown in Fig. 13. It is no surprise that a 4-fold symmetric, constant pitch angle spiral cannot reproduce all the features simultaneously, as there are several examples in the literature of the Milky Way spiral arms deviating from such an idealised form (Taylor \& Cordes 1993; Russeil 2003; Levine et al. 2006). Given the small longitude range covered by the science demonstration field, the loci of such modified spiral arms are to be taken as illustrative only, showing the potential for the complete SEDIGISM survey at high-resolution to provide strong constraints on the positions of the Galactic arms towards the inner Galaxy.

Overall, the assignment of distances to our sample of clouds is broadly consistent with the expected positions of spiral arms, with the exception of the far Norma arm, for which we do not seem to associate many clouds, and the far Sagittarius arm (not shown in Fig. 13), for which we do not find any cloud; the run of this arm is only tentatively known at this location anyway. The small number of clouds associated with the far Norma arm may be due to its close proximity to the more prominent near ScutumCentaurus arm in $l v$ space, and hence the preferred association of clouds with the near distances; or simply because the Norma arm is relatively faint, even at the near distances, making it hardly detectable at the far side. Interestingly the science demonstration field is relatively close to the bar end, and the $3 \mathrm{kpc}$ Arm 

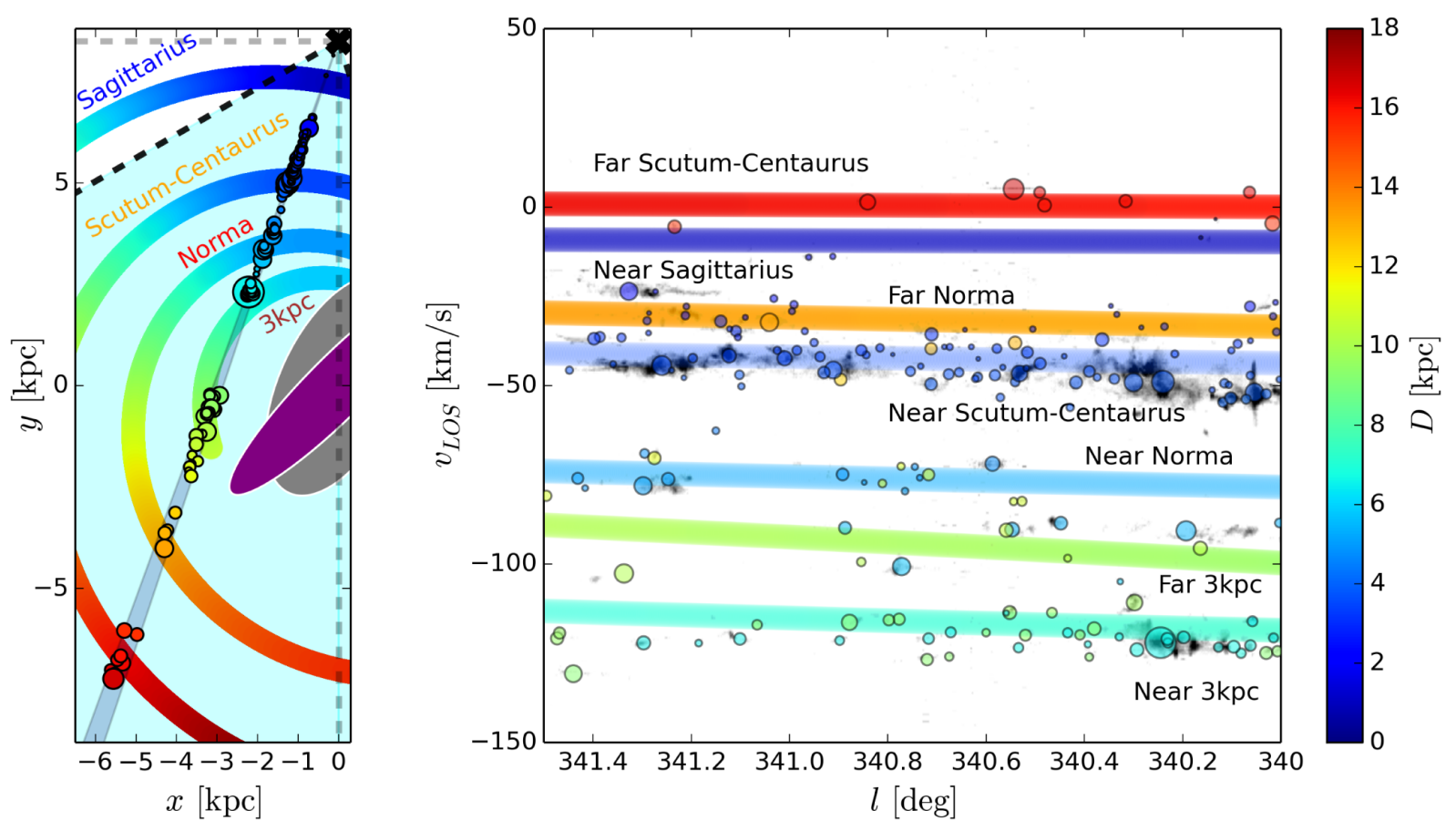

Fig. 13. Left: top-down view of the Galaxy showing the SEDIGISM coverage (full survey in turquoise, and the science demonstration field as the small shaded line of sight). The different spiral arms from our model (see text for details) are shown and labelled with different colours. The positions of all the clouds in the science demonstration field are overplotted as coloured circles, whose colours indicate their assigned distances (see the colour bar to the right), and the size is proportional to the mass of the cloud. Right: $l v$ plot of the peak intensity of ${ }^{13} \mathrm{CO}$ in the science demonstration field (greyscale) overlaid with the positions of all the molecular clouds with assigned velocities (colours and sizes as in the left panel). The positions of the spiral arms are overplotted and labelled, also colour-coded with their distance.

tangency (see Fig. 1); however it is difficult to say with certainty whether such features are seen due to the small longitude extent.

\subsection{Turbulence within GMCs}

Here we show preliminary results of a statistical study of turbulence in the SEDIGISM data. To describe the turbulence, we have applied a Velocity Channel Analysis (VCA) technique, as described in Lazarian \& Pogosyan (2000), on different sections of the ${ }^{13} \mathrm{CO}(2-1) \ell b v$ data cube. This technique consists of computing the spatial power spectra of the two dimensional brightness distribution $\left(I_{2 \mathrm{D}}\right)$ in velocity slices, and letting the thickness $\Delta V$ of the slices vary. As the thickness of velocity slices increases, density fluctuations begin to dominate the emissivity over velocity fluctuations. It is expected that the power spectrum keeps steepening with increasing thickness $\Delta V$ up to a characteristic thickness, above which there is no significant change in the index. When integrating over larger $\Delta V$, most of the velocity fluctuations average out, so that the power spectrum traces only static density fluctuations.

According to turbulence theory, the power spectrum is related with the scale as $P_{I_{N D}}(k) \propto k^{k_{N D}}$, where $N D$ denotes the number of spatial dimensions, and $k$ is the wave number. Values of $k$ go from 1 , the whole length of the longer axis in the data, to $N p i x / 2$, i.e. half the total number of pixels. For incompressible, homogeneous, and isotropic turbulence, $\kappa_{3 D}=-11 / 3$, and $\kappa_{2 D}=-8 / 3$ (Kolmogorov 1941). In the limit of shock-dominated, compressible turbulence, the spatial power index is $\kappa_{3 D}=-4, \kappa_{2 D}=-3$ (Burgers 1974). However, Lazarian \& Pogosyan (2000) predict that the spectral index should saturate to $\kappa_{2 D}=-3$ for an optically thick medium, and many observations support their predictions (cf. Burkhart et al. 2013, who also provide a numerical confirmation for this value of -3 ).

We have applied the VCA method on the six molecular structures extracted with SCIMES with the highest numbers of leaves (Sect. 4 and Table 2), corresponding to some of the most massive and highly sub-structured complexes. The masks generated by SCIMES were used to isolate the ${ }^{13} \mathrm{CO}(2-1)$ brightness distribution of each GMC from the data: new cubes were generated to cover each GMC, and the voxels outside of the masks were set to zero; the size of each cube along each axis was adjusted to the nearest power of two. For our analysis, we let the thickness of the velocity slices vary from twice the velocity resolution $\left(0.5 \mathrm{~km} \mathrm{~s}^{-1}\right)$ up to the thickest slice case, corresponding to integrated maps that include the whole velocity range of the cloud in one channel.

We computed the power spectra as azimuthal median values of the 2D FFT of the data. To properly consider the measured noise, we also calculated the power spectra for emission-free channels. We corrected for the effects of measured noise and beam smearing following the method described in Brunt \& Mac Low (2004). The normalised power spectra for our sample of six GMCs are shown in Fig. 14. We only show the results for angular scales larger than two times the spatial resolution because at smaller scales the effects of the beam size start to dominate. Figure 14 also shows the range over which the least square fitting of the spectral index was performed. Following Medina et al. (2014), the power spectrum fits are made between a minimum scale corresponding to 2.5 times the instrumental resolution, and a maximum scale corresponding to the semi-major axis of each cloud. Given the distances to the various GMCs, the range of scales over which we computed the spectral indices corresponds to linear scales between 1 and $10 \mathrm{pc}$. 

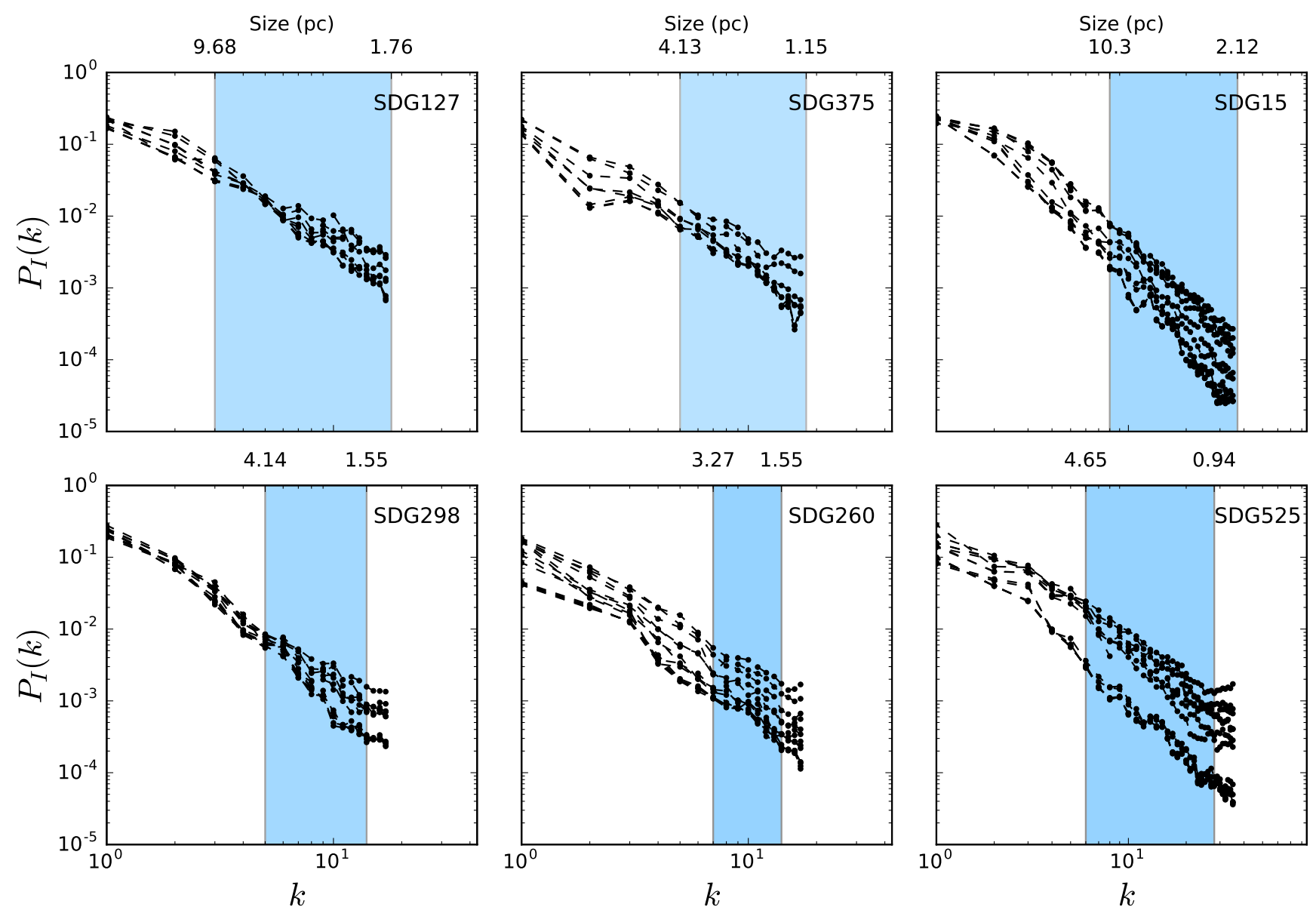

Fig. 14. Normalised spatial power spectra for the six molecular clouds extracted with SCIMES with the highest numbers of leaves, for a range of velocity thickness $\Delta V$ : in each panel, the different curves correspond to increasing thickness from top to bottom, with $\Delta V$ values in the range 0.5 to $18 \mathrm{~km} \mathrm{~s}^{-1}$. The blue area indicates the range of spatial scales over which a least square fitting of the spatial power index was performed. The scale on the lower $X$-axis gives the wave numbers $(k)$, while that on the upper $X$-axis indicates the corresponding scale in pc, using the distance to each GMC, as listed in Table 2. Each panel is labelled with the ID of each GMC (as per Col. 1 in Table 2).

Therefore, this range is well suited to probe turbulence from the scale of a complete cloud, where it may be externally driven (e.g. by supernovea), down to scales where internal sources may contribute to turbulence (e.g. HII regions, stellar winds, proto-stellar outflows; Elmegreen \& Scalo 2004; Dobbs et al. 2014).

In Fig. 15, we show the variations of the spatial power indices $\kappa_{2 \mathrm{D}}$ as a function of velocity slice thickness, $\Delta V$. The spectra become steeper with increasing $\Delta V$, as predicted. Following Lazarian \& Pogosyan (2000) (see also Esquivel et al. 2003), we computed the spectral index for thin slices $\left(\gamma_{\text {thin }}\right)$ as the mean value of all indices corresponding to a thickness $\Delta V<\sigma_{V}$. The thick index $\left(\gamma_{\text {thick }}\right)$ is taken as the average of the indices where $\Delta V>\sigma_{V}$, but restricted to the regime where this index is almost constant. Finally, the index of the second order structure function is computed as $m=2\left(\gamma_{\text {thin }}-\gamma_{\text {thick }}\right)$ in the shallow cases (i.e. where $\left.\gamma_{\text {thick }}>-3\right)$, or $m=2\left(\gamma_{\text {thin }}+3\right)$ in the steep cases (SDG 15 and SDG 298). We find values in the range 0.6-1.8, in rough agreement with the index of the first order structure function derived from principal component analysis $\left(\gamma_{\mathrm{PCA}}\right.$, where $\left.m=2 \times \gamma_{\mathrm{PCA}}\right)$ and published in other studies (e.g. $\gamma_{\mathrm{PCA}} \approx 0.4-$ 0.5, Brunt \& Heyer 2002b; Roman-Duval et al. 2011) ${ }^{6}$. These

\footnotetext{
6 These studies both derived a mean PCA index $\alpha_{\mathrm{PCA}}$ of 0.62 . By rescaling this index to compute the index of the true first order structure function (e.g. Brunt \& Heyer 2002a, 2013; Brunt et al. 2003), we get values in the range $0.4-0.5$.
}

results are based on a very limited sample of six GMCs, and should therefore be regarded with caution. We plan to perform a more systematic analysis on a sample extracted from the full SEDIGISM survey in a forthcoming paper.

Interestingly, all six GMCs have similar characteristic scales of turbulence, corresponding to velocity thickness typically between 4 and $8 \mathrm{~km} \mathrm{~s}^{-1}$, above which velocity fluctuations average out and the spectral indices do not vary much. This could indicate that similar processes are responsible for the turbulence in all clouds in our (small) sample. However, we do find significant variations in the spatial spectral indices between GMCs: two of them (SDG 15 and SDG 298) have steep spectra, with indices that saturate around -3.5 , consistent with the picture of the Kolmogorov energy cascade, and with results reported elsewhere (e.g. Dickey et al. 2001; Muller et al. 2004; see also Elmegreen \& Scalo 2004). The other clouds show spectra that are significantly shallower, with $\kappa_{2 \mathrm{D}}$ between -2.0 and -2.5 for the thick case. According to Lazarian \& Pogosyan (2000), this can be explained by a significant contribution to the turbulence on small scales.

However, we cannot draw robust conclusions from these preliminary results. For example, Burkhart et al. (2013) have shown that the spectral indices also depend on the line excitations. In particular, for a very optically thin, supersonic $\mathrm{CO}$ gas, with low density or low abundance, the spectral index is shallower than the expectations for its column density. Our results are consistent 

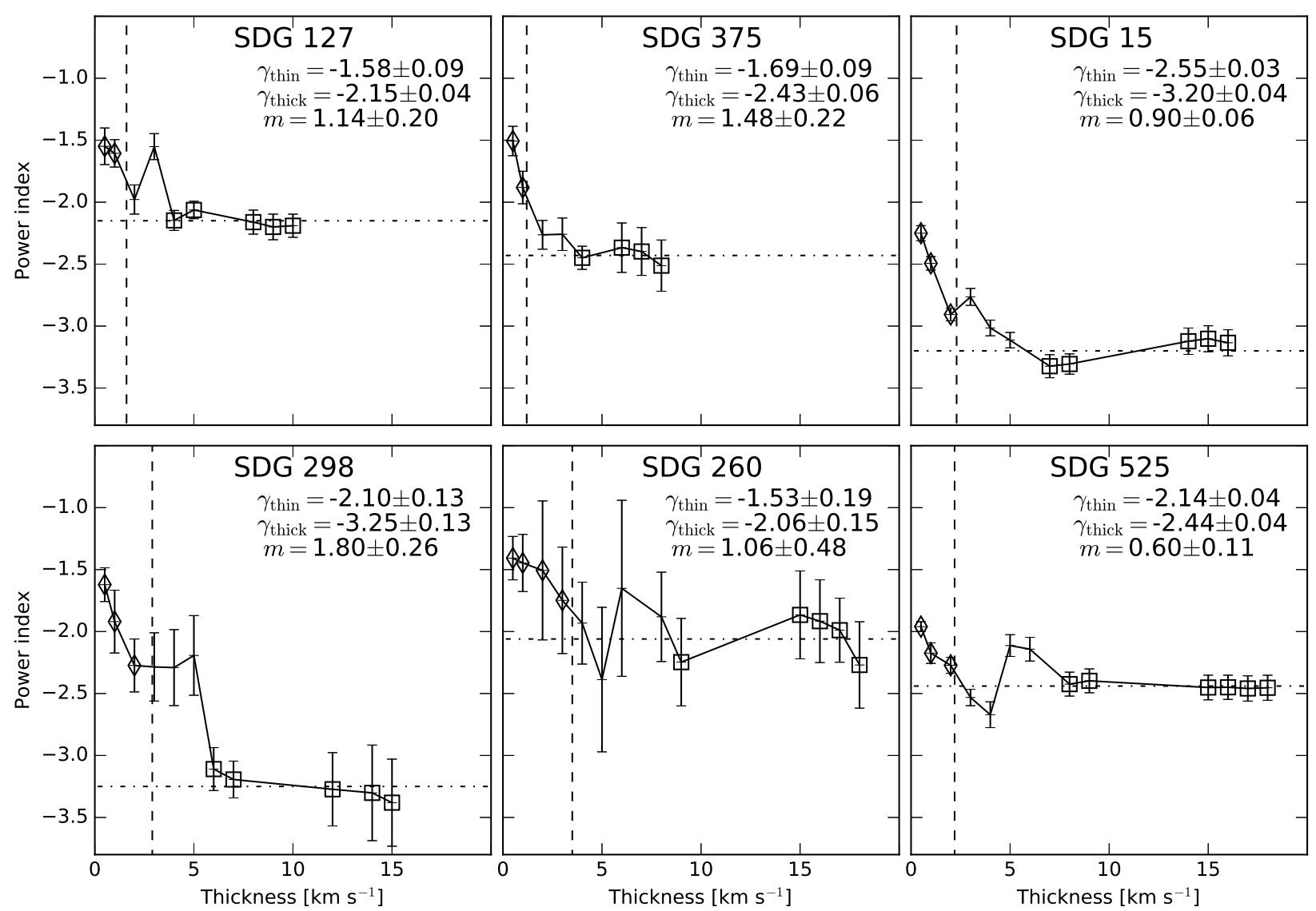

Fig. 15. Variations in the power spectrum indices with velocity thickness for the different GMCs. The error bars correspond to the $1 \sigma$ statistical uncertainties on the fit. The dotted horizontal lines indicate the saturation index for each GMC, corresponding to the thick regime where density fluctuations dominate. The dashed vertical lines show the respective velocity dispersion, $\sigma_{V}$, for each GMC. The data points used to compute the thin and thick indices $\left(\gamma_{\text {thin }}\right.$ and $\left.\gamma_{\text {thick }}\right)$, are indicated with diamonds and square symbols, respectively. These values, as well as the index of the second order structure function $(m)$, are indicated in each panel (see text for details).

with this picture. A more detailed analysis based on a Galaxywide sample of clouds within the full SEDIGISM survey, capable of probing different environments in the Galaxy, will be presented in forthcoming papers. In particular, it will be very interesting to look for possible differences between the Galactic arms, and between the inter- and intra-arm regions.

\section{Dense gas and high-mass star formation}

In order to study the distribution of dense gas within the molecular clouds identified in Sect. 4, and to identify potential sites of high-mass star formation, we have investigated the distribution of compact ATLASGAL sources in the science demonstration field. ATLASGAL has surveyed the inner Galactic plane observing the dust continuum emission at $870 \mu \mathrm{m}$ (Schuller et al. 2009), with a peak flux $1-\sigma$ sensitivity of $\sim 60 \mathrm{mJy} \mathrm{beam}^{-1}$, which corresponds to a column density of $N\left(\mathrm{H}_{2}\right)=1.5 \times$ $10^{21} \mathrm{~cm}^{-2}$ (assuming a dust temperature of $20 \mathrm{~K}$ and absorption coefficient $\left.\kappa_{v}=1.85 \mathrm{~cm}^{2} \mathrm{~g}^{-1}\right)$. ATLASGAL is, therefore, an excellent tracer of the high-density gas within our sample of molecular clouds, potentially pinpointing where high-mass stars are likely to form.

\subsection{Distribution of ATLASGAL clumps}

Within the science demonstration field, there are 140 ATLASGAL clumps from the Compact Source Catalogue (CSC;
Contreras et al. 2013b; Urquhart et al. 2014a). We have made use of the SEDIGISM data to estimate $v_{\text {lsr }}$ for all the clumps, which is essential to place them within their Galactic context, and to associate them with the molecular clouds extracted in Sect. 4. We did so by fitting the ${ }^{13} \mathrm{CO}(2-1)$ spectra towards the peak of the submillimetre emission for all ATLASGAL clumps, using an iterative fitting programme that fits a Gaussian profile to the strongest emission feature, removes the fit from the spectrum, and repeats this process until there is no more emission above three times the rms noise, measured from emission-free channels (see examples presented in Fig. 16).

Although we find multiple components towards $90 \%$ of the sources, in the majority of cases, the integrated intensity of the strongest component is at least twice that of the others and is therefore considered to be the most likely to be associated with the clump, as observed in other studies (e.g. Urquhart et al. 2007). However, for 35 clumps, the multiple components have similar intensities; for these we have either searched the literature for a velocity determined using other high-density tracers, such as $\mathrm{NH}_{3}(1,1)$ or $\mathrm{N}_{2} \mathrm{H}^{+}(1-0)$ (Jackson et al. 2013; Urquhart et al. 2014b; Wienen et al. 2015), or compared the integrated ${ }^{13} \mathrm{CO}(2-1)$ maps of the different velocity components with the ATLASGAL dust emission maps, choosing the velocity component that peaks at the position of the dust emission and where the best correlation between spatial distribution of gas and dust is found (see Fig. 17 for an example of this method). Using this combination of steps we are able to assign a velocity to 



Fig. 16. Example ${ }^{13} \mathrm{CO}$ spectra extracted towards two dense clumps identified from the ATLASGAL survey (black), one with a single velocity component, and another with multiple components along the line of sight. The results of the automatic Gaussian fitting are overlaid in red.



Fig. 17. Integrated ${ }^{13} \mathrm{CO}$ maps of the two components seen towards AGAL340.188-00.411 (see lower panel of Fig. 16). The emission has been integrated over a velocity range of twice the FWHM line width of each component. The yellow contours show the distribution of the $870 \mu \mathrm{m}$ emission mapped by ATLASGAL. In this case, there is a better morphological correlation between the dust emission and the $\mathrm{CO}$ emission found at $-48.9 \mathrm{~km} \mathrm{~s}^{-1}$ than with the $\mathrm{CO}$ emission at $-120.6 \mathrm{~km} \mathrm{~s}^{-1}$. Therefore, we assigned a velocity of $-48.9 \mathrm{~km} \mathrm{~s}^{-1}$ to this clump.

139 clumps in the science demonstration field. For one source (AGAL340.096-00.022), we were unable to identify which velocity component was best representative of the clump.

We have then used the Brand \& Blitz (1993) Galactic rotation model to determine kinematic distances to each of the clumps again solving the kinematic distance ambiguities using the HISA technique (as described in Sect. 4.3), and taking account of their association with IRDCs, and literature information. Three sources are found to be located on the Solar circle (i.e. $\left|v_{\mathrm{lsr}}\right|<10 \mathrm{~km} \mathrm{~s}^{-1}$ ) and so no reliable distance estimate is possible. In total, we have resolved the ambiguities for 97 out of 140 clumps. We have compared our results with those of Wienen et al. (2015) who performed a similar analysis for a
Table 4. Summary of groups identified in the ATLASGAL CSC.

\begin{tabular}{crrrrrc}
\hline \hline $\begin{array}{c}\text { Group } \\
\text { name }\end{array}$ & $N_{\mathrm{cl}} \begin{array}{c}v_{\text {lsr }} \\
\left(\mathrm{km} \mathrm{s}^{-1}\right)\end{array}$ & $\begin{array}{c}\Delta v_{\mathrm{lsr}} \\
\left(\mathrm{km} \mathrm{s}^{-1}\right)\end{array}$ & $\begin{array}{c}d \\
(\mathrm{kpc})\end{array}$ & $\begin{array}{c}\text { SDG } \\
\text { clouds }\end{array}$ & $\begin{array}{c}{ }^{2} \mathrm{CO} \\
\text { GMCs }\end{array}$ \\
\hline G340.249-00.266 & 53 & -49.1 & 3.36 & 3.8 & Multi $^{a}$ & NCEN25 \\
G340.256-00.059 & 16 & -122.5 & 2.22 & 6.7 & Multi $^{b}$ & 3KPC3 \\
G340.529-00.147 & 3 & -46.3 & 0.43 & 3.7 & $331(4)$ & NCEN25 \\
G340.556-00.402 & 2 & -90.1 & 0.46 & 5.6 & $147(4)$ & NORM7.2 \\
G340.749-00.182 & 6 & -36.2 & 2.89 & 3.1 & $430(2)$ & NCEN25 \\
G341.022-00.152 & 2 & -15.2 & 0.54 & 1.5 & $593(1)$ & - \\
G341.034-00.053 & 3 & -38.7 & 3.74 & 3.3 & $408(6)$ & NCEN25 \\
G341.117-00.293 & 29 & -43.0 & 2.78 & 3.5 & Multi $^{c}$ & NCEN25 \\
G341.295+00.336 & 4 & -78.3 & 0.77 & 5.2 & $164(3)$ & NORM8 \\
G341.310+00.209 & 6 & -25.5 & 2.76 & 2.4 & $525(13)$ & NCEN26.5 \\
\hline
\end{tabular}

Notes. ${ }^{(a)}$ SEDIGISM clouds: 234 (5), 260 (9), 273 (2), 298 (7), 323 (2), 324 (1), 392 (2); ${ }^{(b)}$ SEDIGISM clouds: 15 (14), 71 (1); ${ }^{(c)}$ SEDIGISM clouds: 354 (3), 375 (8), 420 (3), 466 (1) . Column 1 is the group name constructed from the mean positions of the associated clumps. Column 2 is the number of associated clumps. Columns 3 and 4 are the mean velocity of the group, and the respective standard deviation (of inter-clump velocities). Column 5 is the adopted distance to the group, i.e. the kinematic distance using the systemic velocity of the group and the respective distance solution. Column 6 is the ID number of the matched molecular clouds identified in Sect. 4 (ID number as listed in Tables 2 and A.1) and the number of leaves associated with them (in parentheses). Column 7 lists the matches to the ${ }^{12} \mathrm{CO}(1-0)$ GMC catalogue presented by García et al. (2014).

sample of 51 clumps located in the science demonstration field and find an overall agreement of $\sim 90 \%$.

Since the ATLASGAL clumps trace the higher density peaks within clouds, it is likely that small groups of ATLASGAL clumps are part of the same larger GMC complex. Identifying these groups can help us to assign distances to clumps for which we have not been able to resolve the distance ambiguity, and to derive more reliable distances by using the systemic velocity of the cloud rather than a number of velocity measurements that may vary over the cloud (cf. Russeil 2003). We have therefore used a friends-of-friends analysis to identify groups of ATLASGAL clumps that are coherent in $\ell b v$ space, allowing a maximum angular offset between adjacent points of 8 arcmins and velocity difference of $8 \mathrm{~km} \mathrm{~s}^{-17}$. This revealed ten coherent groups of sources accounting for 123 of the clumps found in the science demonstration field (see Table 4); this includes 34 clumps that we were unable to resolve the kinematic distance ambiguity for.

Given that the HISA method has a reliability of $\sim 80 \%$ (Busfield et al. 2006; Anderson \& Bania 2009) we would expect some disagreement in the kinematic distance solutions for the ATLASGAL clumps within these larger groups. Indeed, we find this to be the case for the two largest groups, G340.249-00.266 and G341.117-00.293, with 53 and 29 clumps respectively, where $21 \%$ and $14 \%$ of clumps (respectively) had been assigned a far distance, whilst the others had been assigned a near distance. Since these are within the expected fraction of unreliable HISA solutions, we have assigned the near distance to both groups and have therefore revised the distances of the fraction of clumps in disagreement.

García et al. (2014) have also determined distances to six of their lower-resolution ${ }^{12} \mathrm{CO}$ GMCs within the

7 Here we are following the method outlined by Wienen et al. (2015), but, with a more complete set of velocities obtained from the SEDIGISM data, we are able to impose tighter constraints on the position and velocity offsets. 
Table 5. Properties of the ATLASGAL CSC clumps.

\begin{tabular}{|c|c|c|c|c|c|c|c|c|c|}
\hline $\begin{array}{l}\text { ATLASGAL } \\
\text { clump }\end{array}$ & $\begin{array}{c}v_{\mathrm{lsr}} \\
\left(\mathrm{km} \mathrm{s}^{-1}\right)\end{array}$ & $\begin{array}{c}\delta v \\
\left(\mathrm{~km} \mathrm{~s}^{-1}\right)\end{array}$ & $\begin{array}{c}d \\
(\mathrm{kpc})\end{array}$ & $\begin{array}{c}M_{\text {clump }} \\
\left(10^{2} M_{\odot}\right)\end{array}$ & $\begin{array}{c}M_{\mathrm{vir}} \\
\left(10^{2} M_{\odot}\right)\end{array}$ & $\begin{array}{l}\text { SDG } \\
\text { cloud }\end{array}$ & $\begin{array}{l}{ }^{12} \mathrm{CO} \\
\mathrm{GMC}\end{array}$ & $\begin{array}{l}\text { MSF } \\
\text { assoc. }\end{array}$ & $\begin{array}{l}\text { ATLASGAL } \\
\text { group }\end{array}$ \\
\hline AGAL340.119-00.022 & -121.9 & 2.2 & 6.7 & 16.3 & 3.6 & 15 & 3KPC3 & MMB & G340.256-00.059 \\
\hline AGAL340.182-00.047 & -123.7 & 5.3 & 6.7 & 13.8 & 21.4 & 15 & 3KPC3 & MMB & G340.256-00.059 \\
\hline AGAL340.249-00.046 & -121.3 & 5.3 & 6.7 & 76.0 & 53.1 & 15 & 3KPC3 & MMB/HII & G340.256-00.059 \\
\hline AGAL340.359+00.129 & -119.0 & 3.3 & 6.6 & 3.4 & 3.3 & 88 & 3КРC3 & HII & - \\
\hline AGAL340.784-00.097 & -101.4 & 3.9 & 6.0 & 20.0 & 11.7 & 116 & NORM7.4 & MMB & - \\
\hline AGAL340.508-00.442 & -90.5 & 2.8 & 5.6 & 18.8 & 3.8 & 147 & NORM7.2 & - & G340.556-00.402 \\
\hline AGAL340.466-00.299 & -89.3 & 2.6 & 5.7 & 5.9 & 3.7 & 154 & NORM7.2 & HII & - \\
\hline AGAL340.054-00.244 & -53.0 & 8.8 & 3.8 & 43.0 & 87.8 & 234 & NCEN25 & MMB/YSO/HII & G340.249-00.266 \\
\hline AGAL340.248-00.374 & -50.4 & 5.4 & 3.8 & 48.5 & 37.8 & 298 & NCEN25 & MMB/HII & G340.249-00.266 \\
\hline AGAL340.536-00.152 & -46.7 & 3.8 & 3.7 & 8.6 & 11.9 & 331 & NCEN25 & MMB & G340.529-00.147 \\
\hline AGAL340.656-00.236 & -21.7 & 4.2 & 2.1 & 0.4 & 1.7 & -1 & - & MMB & - \\
\hline
\end{tabular}

Notes. Only a small portion of the data is provided here, the full table is available in electronic form at the CDS. Column 1 gives the CSC name. Columns 2 and 3 give the adopted $v_{\mathrm{lsr}}$ and the ${ }^{13} \mathrm{CO}(2-1)$ FWHM line-width. Column 4 gives the distance assigned to the clump. Column 5 is the clump mass, derived from the integrated $870 \mu \mathrm{m}$ flux density. Column 6 is the virial mass, computed as $5 \delta v^{2} R / G$. Columns 7 and 8 list associations with SCIMES clouds (Sect. 4 and Table A.1), and with ${ }^{12} \mathrm{CO}$ clouds from García et al. (2014), respectively. Column 9 indicates matches with HMSF tracers (MMB: methanol maser; HII: compact HII region; YSO: massive young stellar object). Column 10 indicates the ATLASGAL CSC group (see Table 4) to which each clump is associated, if any.

science demonstration field; these are associated with 131 ATLASGAL clumps. Of these, 126 clumps had assigned distances, and we find that 113 (i.e. $~ 90 \%$ ) are in agreement with those assigned by García et al. (2014). We had placed all nine remaining clumps at the far distance, consistent with the fact that all of these are relatively isolated. Given that the five clumps for which we had not solved the distance ambiguity are positionally correlated with GMCs identified by García et al. (2014) we have adopted their distance solution for these sources.

Finally, after cross-matching with the SEDIGISM clouds (as described in Sect. 5.2), we further revised the distances of seven ATLASGAL clumps, within six molecular clouds, to their near distance solutions (as also mentioned in Sect. 4.3). By following these steps we have been able to determine a distance to 136 of the clumps located within the science demonstration field. These adopted distances are listed in Table 5, along with the physical properties of the clumps.

\subsection{Correlation between ATLASGAL groups and GMCs}

As discussed in Sect. 5.1 we have found ten distinct groups of ATLASGAL clumps in the science demonstration field. Comparing these groups with the catalogue of SEDIGISM molecular clouds, we find that the ten ATLASGAL groups correspond to 20 SEDIGISM GMCs, although not all the ATLASGAL clumps in those groups fall within a SEDIGISM cloud. The ID numbers of the matching GMCs are given in Table 4. We also find matches between 15 isolated ATLASGAL clumps and individual molecular clouds, bringing the total number of SEDIGISM clouds with one or more associated ATLASGAL counterparts to 35 (although this includes two clouds within the solar circle, i.e. with $\left|v_{\mathrm{lsr}}\right|<10 \mathrm{~km} \mathrm{~s}^{-1}$ ). The total number of ATLASGAL clumps associated with SEDIGISM GMCs is 129. The exact values of the distances as per the ATLASGAL clump catalogue and the final SEDIGISM cloud catalogue differ typically by less than $\sim 10 \%$ simply because of the variation of exact velocities used for the kinematical distance determination. While this is well within the overall distance uncertainty, for the remainder of the paper we will adopt the distances of the corresponding SEDIGISM clouds for these clumps, for consistency.
In total, there are 11 ATLASGAL clumps not associated with a GMC. One of these does not have a match because we could not assign a velocity. The remaining ten ATLASGAL clumps that do not fall within any of the SEDIGISM clouds are either small (and thus have not passed the criteria of the minimum size required to be part of our cloud catalogue), or, and most often, are in regions where the contrast is too low with respect to their local background (i.e. not above the 4- $\sigma$ requirement to be considered as independent peaks/leaves within the dendrogram); these regions, therefore, form part of a smoother background that did not get assigned to any cloud.

The correlation of multiple SEDIGISM GMCs with a single ATLASGAL group, and the association of a few of the ATLASGAL clumps to a background of more diffuse gas that connects the different GMCs together, tends to confirm our suggestion (Sect. 4.4) that the GMCs identified by SCIMES from the ${ }^{13} \mathrm{CO}$ data are tracing the high-density regions of larger molecular cloud complexes.

\subsection{Dense gas within molecular clouds}

\subsubsection{Mass distribution}

We estimate the isothermal masses of the ATLASGAL clumps using the Hildebrand (1983) method assuming that the total clump mass is proportional to the integrated flux density measured over the source:

$M_{\text {clump }}=\frac{d^{2} S_{v} R}{B_{v}\left(T_{\text {dust }}\right) \kappa_{v}}$,

where $S_{v}$ is the integrated $870 \mu \mathrm{m}$ flux density taken from the ATLASGAL CSC, $d$ is the distance to the source, $R$ is the gasto-dust mass ratio, which we assume to be $100, B_{v}$ is the Planck function for a dust temperature $T_{\text {dust }}$, and $\kappa_{v}$ is the dust absorption coefficient taken as $1.85 \mathrm{~cm}^{2} \mathrm{~g}^{-1}$ (Schuller et al. 2009, and references therein). We use a dust temperature of $20 \mathrm{~K}$, consistent with previous studies in the literature (e.g. Motte et al. 2007; Hill et al. 2005).

In the left panel of Fig. 18 we show the clump mass distribution for all 136 clumps with distances. We also show (in red hatching) the mass distribution for the clumps associated with 

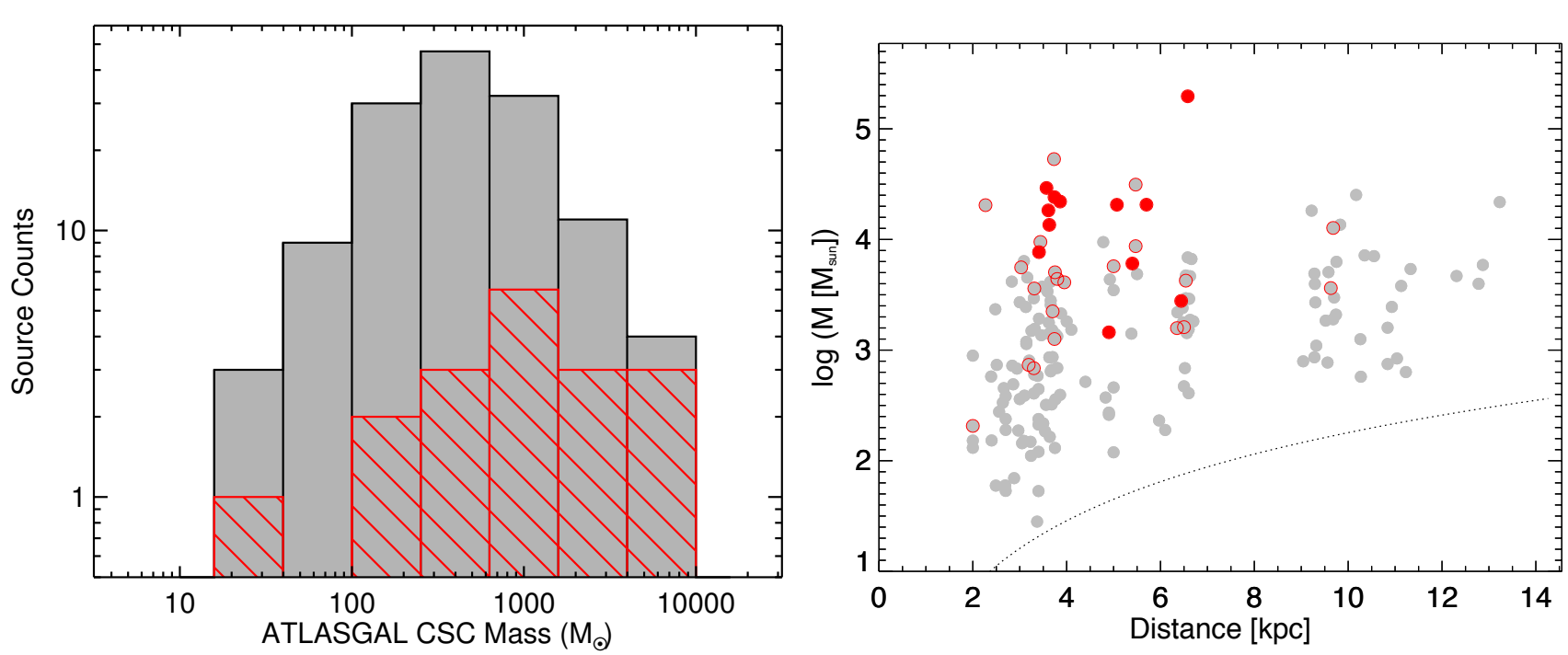

Fig. 18. Left: mass distribution of all ATLASGAL clumps located in the science demonstration field with assigned distances. The mass distribution of clumps associated with a high-mass star formation tracer is shown in red hatching. The bin size is 0.4 dex. Right: mass-distance distribution of all SEDIGISM clouds. Clouds associated with ATLASGAL and with an HMSF tracer are shown in red-filled circles, while the non-HMSF clouds are shown as grey circles (out of which those with an ATLASGAL match are red-outlined). The dotted black line indicates the ${ }^{13} \mathrm{CO}(2-1)$ mass sensitivity limit for an unresolved source (i.e. $28^{\prime \prime}$ radius).

a high-mass star formation (HMSF) tracer from previous studies, such as a methanol maser, a massive young stellar object or a compact HII region (Urquhart et al. 2013, 2014c). Only 18 clumps have been associated with HMSF, and these are mostly associated with methanol masers (14 in total, nine of which have only methanol maser associations, indicating a relatively early stage of their evolution). Three clumps are solely associated with compact HII regions (more evolved), four clumps are associated with two different tracers, and two clumps have all three tracers, hosting multiple evolutionary stages. In the right panel of Fig. 18 we show the SEDIGISM cloud mass distribution as a function of distance, where grey-filled circles show clouds without a known HMSF tracer, grey circles with red outline show clouds with at least one ATLASGAL clump but no HMSF tracer, and red-filled circles show clouds with an ATLASGAL and an HMSF tracer.

Although the statistics are low, there is a trend for the HMSF regions to be in the most massive clumps, within the most massive clouds. We note that a larger proportion of the most massive clumps are associated with star formation (Urquhart et al. 2014b) and that these tend to be warmer than more quiescent clumps (e.g. Urquhart et al. 2011; Wienen et al. 2012). This may lead to clump masses being over estimated; however, at the angular scales of the structures probed here (i.e. $~ 30^{\prime \prime}$ ) the temperature range from starless clumps to those hosting HII regions or photo-dissociation regions is 15 to $25 \mathrm{~K}$ (Dunham et al. 2011; Urquhart et al. 2011; Wienen et al. 2012; Deharveng et al. 2015; Guzmán et al. 2015). A difference of $\pm 5 \mathrm{~K}$ around the temperature used to estimate the clump masses corresponds to a maximum variation of approximately $30 \%$ in the dust masses and so does not have a significant impact on the overall clump mass distribution.

\subsubsection{Dense gas fraction}

In order to investigate whether the presence of high-mass star formation was related to the amount of dense gas within each cloud, we determined the dense gas fraction (DGF) of the
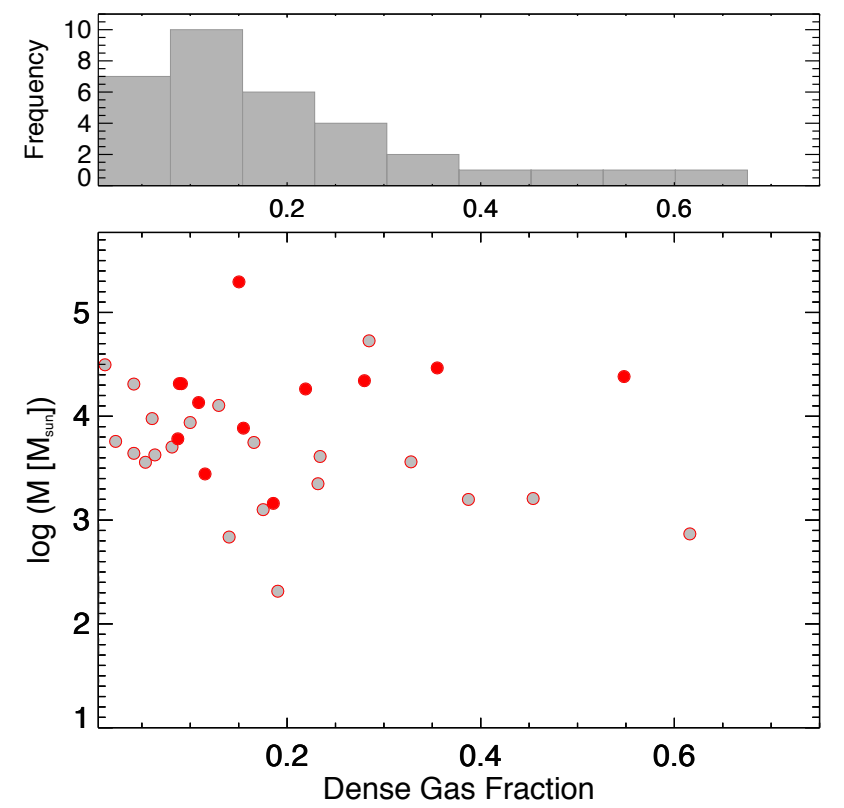

Fig. 19. Top: histogram of the dense gas fraction (DGF) of the GMCs associated with ATLASGAL clumps. Bottom: distribution of the DGF as a function of cloud mass for all GMCs that have an ATLASGAL counterpart, colour-coded according to the existence of an HMSF marker (red for clouds with an HMSF tracer, and grey for no HMSF).

\section{SEDIGISM GMCs using:}

$\mathrm{DGF}=M_{\mathrm{clump}} / M_{\mathrm{GMC}}$,

where $M_{\text {clump }}$ is the clump mass, as defined above. When several clumps are associated with a single GMC, the masses of all the clumps are summed together. We assume that the dust emission is tracing the dense gas at column densities above $\sim 7.5 \times 10^{21} \mathrm{~cm}^{-2}$, corresponding to the 5- $\sigma$ limiting sensitivity for ATLASGAL.

In Fig. 19 we show the histogram of the dense gas fraction of the GMCs, and the distribution of DGF as a function of cloud 

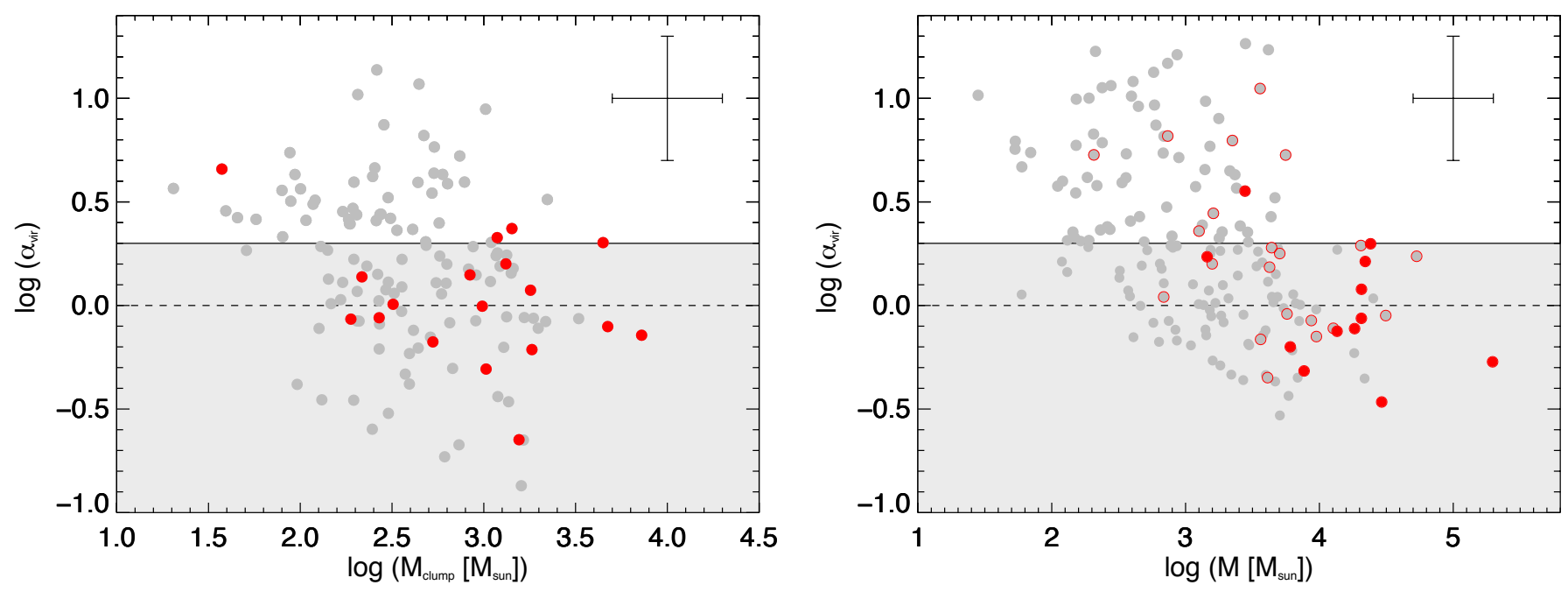

Fig. 20. Virial parameter $\left(\alpha_{\text {vir }}\right)$ as a function of clump mass $\left(M_{\text {clump }}\right)$ in the left panel, and cloud mass $(M)$ in the right panel. This is shown for the HMSF and non-HMSF sub-samples; these are indicated as red and grey circles, respectively. In the right panel, GMCs with an ATLASGAL match but no HMSF tracer are shown as grey circles with a red outline. The solid horizontal line indicates the critical value of $\alpha_{\text {vir }}=2$, for an isothermal sphere in hydrostatic equilibrium without magnetic support. The dashed black line indicates an $\alpha_{\mathrm{vir}}=1$. The light grey shading indicates the region where clouds are unstable and likely to be collapsing without additional support from a strong magnetic field. Representative error bars, corresponding to a factor two uncertainty, are shown in the upper-right corner of each panel.

mass. The median value of DGF for the clouds with an ATLASGAL counterpart is $\sim 15 \%$ (and a mean value of $\sim 21 \%$ ), which is 2-3 times higher than reported in the literature (i.e. 2-7\%, cf. Eden et al. 2013, Ragan et al. 2014, Battisti \& Heyer 2014, Csengeri et al. 2016). However, this represents only a small fraction of the clouds extracted with SCIMES (33 out of 182). The remaining ones would have a DGF close to zero, so that the mean DGF value would be significantly lower when including all clouds $(\sim 4 \%)$. In addition, the ${ }^{13} \mathrm{CO}(2-1)$ transition has a higher critical density than ${ }^{12} \mathrm{CO}(1-0)$, which was used in some of these previous studies, and is therefore not necessarily capturing all of the material associated with the extended envelope. The SEDIGISM ${ }^{13} \mathrm{CO}(2-1)$ data have a column density sensitivity of a few $10^{21} \mathrm{~cm}^{-2}$ and, as a consequence, the DGFs estimated are likely to be upper limits to the true values. Finally, one could expect that for clouds with angular sizes smaller or similar to the maximum recoverable scale in the ATLASGAL data (uniform emission on scales larger than $\sim 2.5^{\prime}$ is filtered out during data reduction, Schuller et al. 2009), the signal detected by the bolometers could encompass most of the cloud rather than simply the denser parts (see e.g. the discussion in Battisti \& Heyer 2014). This would also result in over estimating the true DGF. We investigated this effect in our sample, and we found that clouds with small angular sizes have a wide range of DGFs (between 0 and $60 \%$ ), but also that some well resolved clouds, with angular sizes an order of magnitude larger than the maximum recoverable scale of ATLASGAL, have a relatively large DGF (up to $\sim 60 \%$ ). Therefore, we do not think that this effect is significant in our sample.

We do not find any particular trend of DGF with the cloud masses nor with the existence of high-mass star formation. Instead, the existence of HMSF seems to correlate better with the total mass of the cloud. We caution that the statistics within the SEDIGISM science demonstration field are low for any firm conclusions, and therefore, this will be further investigated in a subsequent paper using the complete survey.

\subsubsection{Virial parameter}

Finally, we estimated the virial parameters for all clumps using the same formula described in Sect. 4.4 and used this to evaluate their stability. In the left panel of Fig. 20 we show the virial parameters as a function of clump mass. There is a clear trend for decreasing virial parameters with increasing mass, indicating that the most massive clumps are also the most gravitationally unstable, and the more likely to be undergoing collapse unless they are supported by strong magnetic fields. Similar trends have been reported in the literature (e.g. Barnes et al. 2011, Kauffmann et al. 2013, Urquhart et al. 2015). We note that all of the HMSF clumps are found in the unstable part of the parameter space, which would suggest that, even if magnetic fields can stabilise the clumps globally (e.g. Pillai et al. 2015), they are unlikely to be able to do this on smaller size scales, as star formation is clearly ongoing in some of these clumps.

Interestingly, if we plot the virial parameter of the SEDIGISM clouds as a function of cloud mass, we find a similar trend as for the clumps (Fig. 20, right panel), even though we have more clouds in the unbound regime, as a consequence of the lower surface densities traced by ${ }^{13} \mathrm{CO}(2-1)$. Despite that, this figure shows that most clouds that are unstable on clump scales, and most particularly, the clouds that already show signs for HMSF, do seem to be gravitationally unstable even at the larger scales of the GMCs, in line with the idea that HMSF is preferentially taking place in globally collapsing clouds (e.g. Barnes et al. 2010; Schneider et al. 2010; Peretto et al. 2013). A similar trend for the most massive clouds to appear more likely to be bound is also seen in simulations (e.g. Dobbs et al. 2011).

\section{Excitation, optical depth, and physical conditions}

\subsection{Excitation and column density}

The two CO isotopologues' $J=2-1$ emission lines observed with SEDIGISM, in particular when combined with the three 
iso-CO $J=1-0$ lines from ThrUMMS (Barnes et al. 2015), allow us to derive a detailed, spatially- and velocity-resolved distribution for various physical and chemical properties in all the observed molecular clouds, including: optical depths, excitation temperatures, molecular abundances, and column densities. Barnes et al. (2015) already demonstrated the diagnostic power of such an approach with the ThrUMMS data alone, finding a new value of the conversion factor between $\mathrm{CO}$ emissivity and mass column density, which suggests that the total molecular mass of the Milky Way may have previously been substantially underestimated. They did this assuming only a common LTE excitation between the three main iso-CO species, and a fixed intrinsic abundance ratio $R_{13}=\left[{ }^{12} \mathrm{CO}\right] /\left[{ }^{13} \mathrm{CO}\right]$. While the latter may indeed also vary, their results on the mass distribution are relatively insensitive to the exact value assumed for $R_{13}$.

The validity of a common $T_{\mathrm{ex}}$ between the very optically thick ${ }^{12} \mathrm{CO}$ lines and the more typically optically thin ${ }^{13} \mathrm{CO}$ and $\mathrm{C}^{18} \mathrm{O}$ lines is a more relevant issue, but the SEDIGISM data now allow a straightforward resolution to this issue as well. Building upon the method described by Kramer et al. (1999) and Hernandez et al. (2011), we have developed a root-finding algorithm to compute $\tau, T_{\mathrm{ex}}$ and the column density at each voxel of the data cube. We arrive at our solutions by matching the column density calculated from each ${ }^{13} \mathrm{CO}$ transition across a range of $T_{\mathrm{ex}}$. The column density is given by the usual plane-parallel radiative transfer equation:

$N=\frac{3 h}{8 \pi^{3} \mu^{2}} \frac{Q\left(T_{\text {ex }}\right) \mathrm{e}^{E_{l} / k T_{\text {ex }}}}{J_{u}\left(1-\mathrm{e}^{-h v / k T_{\text {ex }}}\right)} \int \tau_{u l} \mathrm{~d} V$,

where the total $N$ is calculated separately for each transition line $\left(J_{u}=2\right.$ or 1$), \mu$ is the dipole moment of the CO molecules, $Q\left(T_{\mathrm{ex}}\right)$ is the rotational partition function, and $E_{\mathrm{l}}$ is the energy of the lower state of transition $J_{u} \rightarrow J_{u}-1$.

The optical depth, $\tau_{u l}$, is derived through the plane-parallel radiative transfer equation:

$T_{\mathrm{mb}}=\frac{h v}{k}\left(f_{T_{\mathrm{ex}}}-f_{T_{\mathrm{bg}}}\right)\left(1-\mathrm{e}^{-\tau_{u l}}\right)$.

Here $T_{\mathrm{mb}}$ is the main beam brightness temperature, $T_{\mathrm{bg}}$ is the background temperature of $2.73 \mathrm{~K}$, and $f_{T}=[\exp (h v /(k T))-$ $1]^{-1}$. Since $T_{\mathrm{mb}}$ for both transitions are observed with either the ThrUMMS or SEDIGISM surveys, we can use Eqs. (4) and (5) to express the ratio between the $J=2-1$ and $J=1-0$ column densities as a function of $T_{\mathrm{ex}}$ :

$\eta_{21}\left(T_{\mathrm{ex}}\right)=\left|\log \left(\frac{N_{\mathrm{tot}, 21}}{N_{\mathrm{tot}, 10}}\right)\right|$,

where $N_{\text {tot,21 }}$ and $N_{\text {tot,10 }}$ are the total column densities calculated from each line transition using Eq. (4).

To improve computing time we simplified the iterative method of Hernandez et al. (2011), who estimated the threedimensional $T_{\text {ex }}$ distribution throughout a highly filamentary IRDC using $\mathrm{C}^{18} \mathrm{O} J=2-1$ and $\mathrm{C}^{18} \mathrm{O} J=1-0$. This modification is possible since Eq. (6) is a function with a global minimum within a domain of $T_{\mathrm{ex}} \geq 2.73 \mathrm{~K}$, which represents when $N_{21}$ and $N_{10}$ are equal (i.e. when $\eta_{21}=0$ ). Thus, the voxel $T_{\text {ex }}$ can be estimated by simply minimizing $\eta_{21}$ within $T_{\text {ex }}$ of range 2.73 to $30 \mathrm{~K}$, the typical excitation temperature range for GMCs (e.g. Barnes et al. 2015). By equating $N_{21}$ and $N_{10}$, we are assuming that their excitation temperatures are equal. It is possible that the excitation will differ between the two transitions and produce unequal, possibly sub-thermal, excitation temperatures.



Fig. 21. Top: total ${ }^{13} \mathrm{CO}$ column density as estimated over a range of $T_{\mathrm{ex}}$ from each line transition separately using Eq. (4). Bottom: distribution of $\eta_{21}$ (Eq. (6)) over the same range of $T_{\text {ex }}$. Since we have defined $\eta_{21}$ as the absolute difference between the two total column density estimates, the $T_{\text {ex }}$ in this voxel is determined by locating the global minimum, as shown by the vertical dotted black line. For this example voxel, we find $T_{\mathrm{ex}}=7.8 \mathrm{~K}$.

However, for lower density cloud regions, Jiménez-Serra et al. (2010) combined ${ }^{13} \mathrm{CO} J=1-0$ data with the $J=3-2$ and $J=2-1$ LVG analysis, finding that the overall column densities were within a factor of 2 . We find that most voxels with $S / N>\sim 4$ have line ratios that allow convergence to a single assumed $T_{\text {ex }}$. For some noisier voxels, the algorithm fails to converge to a $T_{\mathrm{ex}}$ solution due to "unphysical" line ratios assuming a shared $T_{\mathrm{ex}}$; these voxels are then omitted from our analysis. While mathematically this might suggest differential thermalisation, we discount this possibility due to the low $\mathrm{S} / \mathrm{N}$ at these locations.

For the present study, we use the ${ }^{13} \mathrm{CO}$ data from both surveys. We first convolve the $30^{\prime \prime}$ resolution SEDIGISM cubes to the $72^{\prime \prime}$ resolution of the ThrUMMS data. $T_{\mathrm{ex}}$ was estimated for all voxels with $T_{\mathrm{mb}}$ measurement above zero to avoid unphysical column density estimates and improve computing time. Figure 21 presents the $T_{\text {ex }}$ solution for one example voxel. For each voxel with a $T_{\mathrm{ex}}$ solution, we are able to compute the opacities $\left(\tau_{21}\right.$ and $\left.\tau_{10}\right)$ and total column density, $N\left({ }^{13} \mathrm{CO}\right)$. Finally, by performing this analysis for each voxel, we are able to derive the three-dimensional spatially- and velocity-resolved distribution of the physical conditions of the ${ }^{13} \mathrm{CO}$ gas.

Figure 22 presents the results for $\tau_{2-1}, T_{\mathrm{ex}}$ and $N\left({ }^{13} \mathrm{CO}\right)$ in the science demonstration field, as longitude-velocity maps integrated along $b$. The distributions of these three quantities on a voxel basis are shown in Fig. 23. Interestingly, the $T_{\mathrm{ex}}$ and $\tau$ distributions, while each contributing to the column density, are distinctly different in several places. That is, some locations with high $N$ are mostly due to a high excitation while other locations derive their high $N$ from a high opacity. The latter is especially interesting since we see that the highest column density clumps reach peak ${ }^{13} \mathrm{CO}$ opacities of $\sim 8$, which certainly shows that common assumptions about optically thin emission can lead one's analysis and physical interpretation astray. Maps of single lines cannot by themselves give us this physical insight. 

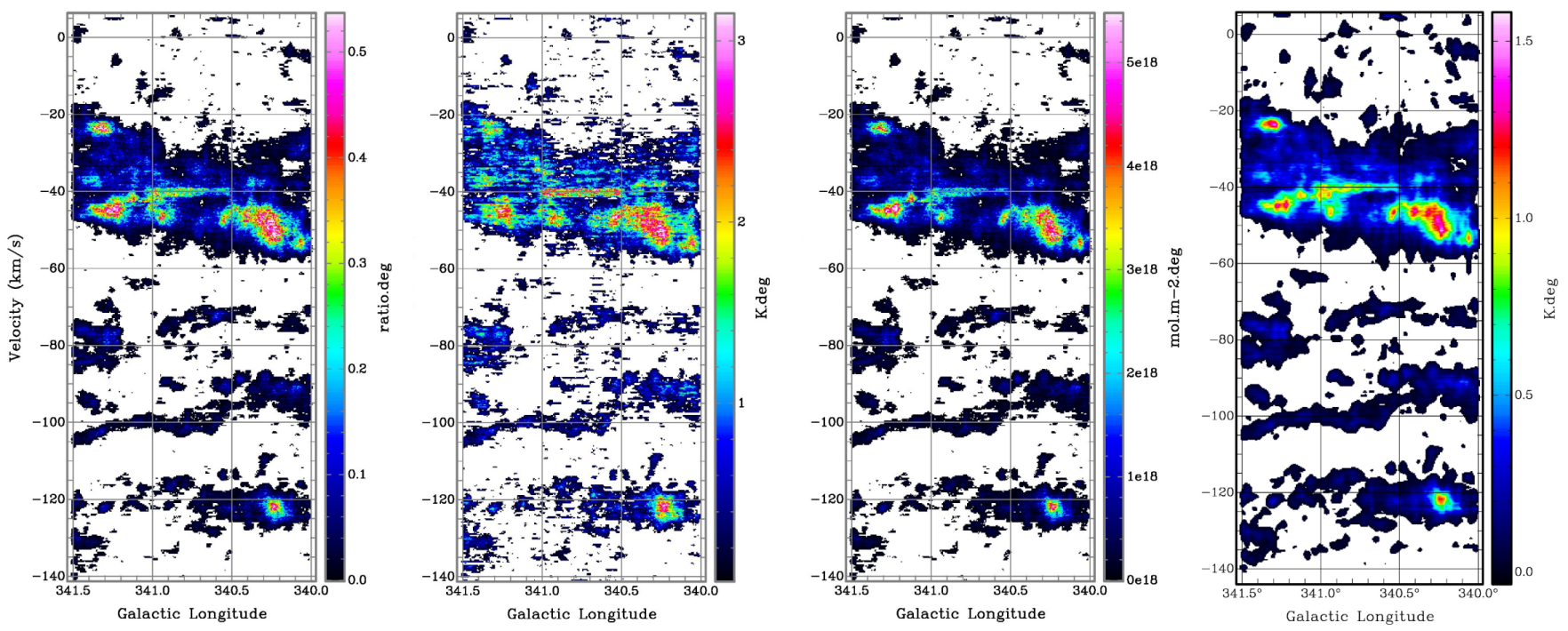

Fig. 22. Radiative transfer solutions, obtained as described in Sect. 6.1, for $\tau(2-1)$ (left panel) and $T_{\mathrm{ex}}$ (second panel) of the ${ }^{13} \mathrm{CO}$ emission data cubes. Third panel: resulting column density $N\left({ }^{13} \mathrm{CO}\right)$, derived from the optical depth and excitation temperature cubes. The data in each panel have been integrated over the 1-degree extent in latitude, including any zero-valued pixels, to form the respective longitude-velocity moment maps shown here. Right panel: shows a similar $l v$ moment map of the ${ }^{13} \mathrm{CO}(2-1)$ emission as seen in the SEDIGISM data convolved to the ThrUMMS resolution.
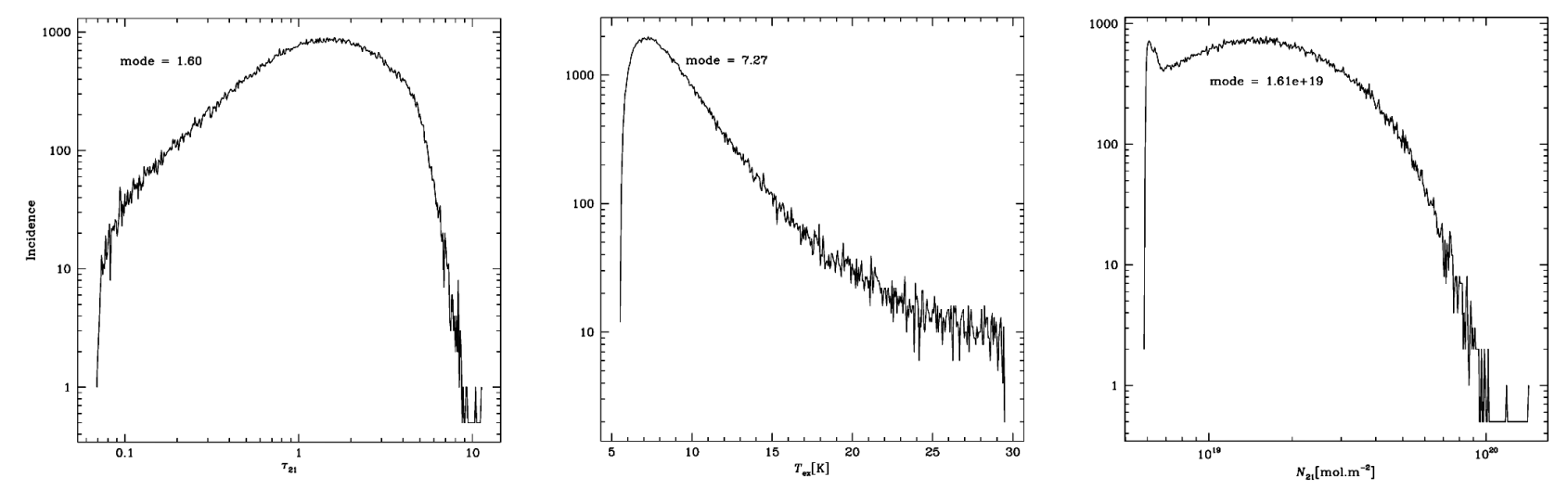

Fig. 23. Distributions of $\tau(2-1)$ (left panel), $T_{\mathrm{ex}}$ (middle panel) and $N\left({ }^{13} \mathrm{CO}\right)$ (right panel) for the 140000 usable voxels in the science demonstration field.

\subsection{The ${ }^{13} \mathrm{CO} X$-factor}

From this radiative transfer solution, we can directly compute an important result which bears on much of the new science presented here, as well as confirming previously published results. As shown by Barnes et al. (2015), a voxel-by-voxel calculation of the ratio of ${ }^{13} \mathrm{CO}$ column density to integrated intensity, $N / I$, can be used as a direct probe of the spatially-resolved $X$-factor, relating integrated intensity (here for ${ }^{13} \mathrm{CO}$, but usually for ${ }^{12} \mathrm{CO}$ in the literature) to the total molecular hydrogen column density. When this ratio is plotted as a function of $I$, one can also reveal the nature of the conversion law, that is whether it is "flat" (constant $X$, the standard method for many decades) or a more complex function of other parameters (e.g. Narayanan et al. 2012, Barnes et al. 2015).

We present this comparison here in Fig. 24. Unlike the result for the ${ }^{12} \mathrm{CO}(1-0)$ line $\left(X \propto I^{0.4}\right.$, Barnes et al. 2015), we see that $X$ is statistically flat for ${ }^{13} \mathrm{CO}(2-1)$ with a mean value of $1.8 \times 10^{15} \mathrm{~cm}^{-2}\left(\mathrm{~K} \mathrm{~km} \mathrm{~s}^{-1}\right)^{-1}$ across the 140,000 voxels in the science demonstration field with $I\left({ }^{13} \mathrm{CO}\right)>5-\sigma$.

To convert this to a true $X$-factor for the ${ }^{13} \mathrm{CO}(2-1)$ line, we need to multiply this mean by two gas-phase abundances, $\left[{ }^{12} \mathrm{CO}\right] /\left[{ }^{13} \mathrm{CO}\right]$ and $\left[\mathrm{H}_{2}\right] /\left[{ }^{12} \mathrm{CO}\right]$. For simplicity, we take the first ratio as 60 to conform with Barnes et al. (2015), and the second as $10^{4}$ (e.g. Dame et al. 2001, Bolatto et al. 2013). Then we obtain a mean $X$ factor in the science demonstration field, based only on the ${ }^{13} \mathrm{CO}$ data from the ThrUMMS and SEDIGISM surveys, of $1.08 \pm 0.19 \times 10^{21} \mathrm{~cm}^{-2}\left(\mathrm{~K} \mathrm{~km} \mathrm{~s}^{-1}\right)^{-1}$.

This result agrees very well with the factor derived from the comparison between Hi-GAL and ${ }^{13} \mathrm{CO}(2-1)$ data (see Sect. 4.4). Although fortuitous to some extent, based on the inclusion of somewhat uncertain conversion parameters in both estimates, this close agreement is remarkable. In addition, since we have used the same approach as in Barnes et al. (2015) to compute the ${ }^{13} \mathrm{CO}$ column density, the above agreement in $X$ estimates gives strong confirmation to the ThrUMMS ${ }^{12} \mathrm{CO}$ conversion law as well. In particular, the clear difference in the behaviour of the conversion law for ${ }^{13} \mathrm{CO}$ (flat) compared to ${ }^{12} \mathrm{CO}$ (a function of $I\left({ }^{12} \mathrm{CO}\right)$ at least) lends credence to Barnes et al.'s (2015) argument that their ${ }^{12} \mathrm{CO}$ conversion law arises from the extremely high opacity in the ${ }^{12} \mathrm{CO}(1-0)$ line ( $\tau$ up to $\sim 400$ ).

A direct implication of our approach is that we will be able to make reliable maps of the total molecular column density, from a combination of SEDIGISM and ThrUMMS data, across 




Fig. 24. Ratio of column density from radiative transfer computation to ${ }^{13} \mathrm{CO}(2-1)$ integrated intensity, $N / I$, as a function of the same integrated intensity, for the $\sim 140000$ voxels in the science demonstration field with $I$ above a $5-\sigma$ level of $0.44 \mathrm{~K} \mathrm{~km} \mathrm{~s}^{-1}$. The red line shows the trend of median values (which are very similar to the means) for the data binned into ten equal $\log (I)$ intervals while the green lines show the 2- $\sigma$ excursions from the median values.

the entire $10^{9}$ voxel data set of these surveys. With distances to the various kinematic features, these column densities are then readily converted into masses of individual clouds, as identified e.g. by the SCIMES algorithm in Sect. 4. Also, combining these results with the $\mathrm{C}^{18} \mathrm{O}$ SEDIGISM data and the $J=1-0$ ${ }^{12} \mathrm{CO}$ data from ThrUMMS will further allow us to construct spatially- and velocity-resolved maps of the various molecular abundances, and relate any abundance variations we may see to environmental or other factors.

\section{Filamentary structures}

In this section, we investigate the presence of filamentary structures in the science demonstration field and outline the potential of SEDIGISM to verify their coherence in velocity, and derive their properties: velocity dispersion, length, column density, mass, and linear mass density. In Sect. 7.1, we discuss the filament candidates identified in the ATLASGAL and Hi-GAL continuum surveys (Schuller et al. 2009; Molinari et al. 2010); in Sect.7.2, we show the results of applying the DisPerSE algorithm (Sousbie 2011) directly on the ${ }^{13} \mathrm{CO}$ data cube.

\subsection{Filament candidates in ATLASGAL and Hi-GAL}

Recently, the ATLASGAL and Hi-GAL surveys were used to identify filament candidates in the Galactic plane through the analysis of their continuum emission at $870 \mu \mathrm{m}$ ( $\mathrm{Li}$ et al. 2016), and at 70, 160, 250, 350 and $500 \mu \mathrm{m}$ (Schisano et al. 2014; Wang et al. 2015), respectively. The two surveys deliver a unique dataset to compile an unbiased catalogue of filament candidates throughout the Galaxy. Indeed, ATLASGAL provides high angular resolution at a wavelength sensitive to the cold dust and is unaffected by background contamination and saturation, which complicate the analysis of Hi-GAL data. On the other hand,



Fig. 25. Integrated intensity map of the ${ }^{13} \mathrm{CO}(2-1)$ (top) and $\mathrm{C}^{18} \mathrm{O}(2-1)$ (bottom) transitions towards G341.246-0.267 in the velocity range of $[-48.2,-41.0] \mathrm{km} \mathrm{s}^{-1}$. Grey levels are from $10-\sigma\left(9.4 \mathrm{~K} \mathrm{~km} \mathrm{~s}^{-1}\right)$ for ${ }^{13} \mathrm{CO}$ in steps of $10-\sigma$, and from $5-\sigma\left(4.7 \mathrm{~K} \mathrm{~km} \mathrm{~s}^{-1}\right)$ for $\mathrm{C}^{18} \mathrm{O}$ in steps of 5- $\sigma$. The blue solid line marks the main spine of the filament identified; the magenta lines mark the other sub-branches identified on the ATLASGAL dust emission map by Li et al. (2016). The yellow empty circles mark the positions where the six spectra shown in Fig. 26 are extracted. The red thin line marks the dilation box used to compute the length and the mass of the filament.

Hi-GAL is more sensitive to emission from low-density structures, down to values of $\sim 10^{21} \mathrm{~cm}^{-2}$ at $16 \mathrm{~K}$, while ATLASGAL has a $5-\sigma$ column density sensitivity of $\sim 7.5 \times 10^{21} \mathrm{~cm}^{-2}$ for a dust temperature of $20 \mathrm{~K}$.

Li et al. (2016) identified twelve filamentary structure candidates in the SEDIGISM science demonstration field based on the ATLASGAL data, nine of which are single filament candidates (elongated linear structures with typical aspect ratios larger than three), and the other three being networks of filaments (several filaments that seem to be connected to each other). A study of Hi-GAL column density map of the region reveals 88 filament candidates. Details on the two catalogues and on the methods used to identify the structures and extract their dust properties are given by Li et al. (2016), Schisano et al. (2014) and Schisano et al. (in prep.). All twelve structures detected in ATLASGAL are also found in the Hi-GAL sample, hence, in the following discussion, we will focus on this common candidate list.

As a first step, we verified the coherence in velocity of the filament candidates making use of the SEDIGISM ${ }^{13} \mathrm{CO}(2-1)$ and $\mathrm{C}^{18} \mathrm{O}(2-1)$ data. For this purpose, we extracted spectra along the skeleton of each filament (i.e. the centre positions of each filament as identified by Li et al. 2016), and we analysed the position-velocity diagrams. We then averaged the spectra in a dilation box of width equal to three SEDIGISM beams (see Fig. 25) to derive the central velocity and the width of the detected spectral features. Finally, we computed integrated 
Table 6. Catalogue of ATLASGAL filaments (top) and networks (bottom) in the science demonstration field.

\begin{tabular}{|c|c|c|c|c|c|c|c|c|c|c|}
\hline Name & $\begin{array}{c}v \\
\left(\mathrm{~km} \mathrm{~s}^{-1}\right)\end{array}$ & $\begin{array}{c}\Delta v \\
\left(\mathrm{~km} \mathrm{~s}^{-1}\right)\end{array}$ & 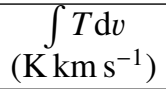 & $\begin{array}{c}A \\
\left(\mathrm{pc}^{2}\right)\end{array}$ & $\begin{array}{c}d \\
(\mathrm{kpc})\end{array}$ & $\begin{array}{c}M_{13} \mathrm{CO} \\
\left(10^{3} M_{\odot}\right)\end{array}$ & $\begin{array}{c}l \\
(\mathrm{pc})\end{array}$ & $\begin{array}{c}\sigma_{v} \\
\left(\mathrm{~km} \mathrm{~s}^{-1}\right)\end{array}$ & $\begin{array}{l}(M / l)_{\mathrm{obs}} \\
\left(M_{\odot} / \mathrm{pc}\right)\end{array}$ & $\begin{array}{l}(M / l)_{\text {vir }} \\
\left(M_{\odot} / \mathrm{pc}\right)\end{array}$ \\
\hline \multicolumn{11}{|c|}{ Filaments } \\
\hline G340.301-00.387 & -49.3 & $-57.2,-41.4$ & 22.18 & 45.82 & 3.8 & $28.0^{\dagger}$ & 23.61 & 1.56 & 1187 & 1127 \\
\hline G340.316+00.079 & -111.4 & $-116.5 /-106.3$ & 5.59 & 39.63 & 6.2 & 4.7 & 11.15 & 0.51 & 424 & 121 \\
\hline G340.482-00.306 & -45.0 & $-51.3 /-38.7$ & 9.54 & 15.16 & 3.8 & $4.5^{\dagger}$ & 7.08 & 1.40 & 635 & 909 \\
\hline G340.511-00.471 & -43.5 & $-50.2 /-36.8$ & 17.50 & 11.33 & 3.5 & 4.4 & 5.58 & 1.08 & 785 & 546 \\
\hline G340.981-00.013 & -46.9 & $-50.3 /-43.5$ & 7.11 & 19.40 & 3.4 & 3.1 & 11.42 & 1.27 & 272 & 750 \\
\hline G341.244-00.265 & -44.6 & $-48.2 /-41.0$ & 17.43 & 39.81 & 3.6 & 22.4 & 21.34 & 1.04 & 1049 & 507 \\
\hline G341.415+00.244 & -37.4 & $-39.6 /-35.2$ & 8.27 & 7.17 & 3.2 & 1.3 & 4.26 & 0.83 & 312 & 320 \\
\hline \multicolumn{11}{|c|}{ Networks } \\
\hline G340.200-00.035 & -122.2 & $-127.3 /-117.1$ & 22.70 & 116.52 & 6.6 & 59.2 & & & & \\
\hline G340.236-00.153 & -50.9 & $-58.3 /-43.5$ & 18.55 & 209.95 & 3.8 & 122.0 & & & & \\
\hline G340.941-00.319 & -45.9 & $-50.4 /-51.4$ & 10.37 & 74.70 & 3.6 & 30.1 & & & & \\
\hline G341.306+00.339 & -78.3 & $-82.3 /-74.3$ & 12.71 & 56.84 & 5.2 & 16.0 & & & & \\
\hline
\end{tabular}

Notes. ${ }^{(\dagger)}$ For G340.301-00.387 and G340.482-00.306, the masses from the ${ }^{13} \mathrm{CO}$ data are likely overestimated by up to $20 \%$ and $34 \%$, respectively, due to contamination from emission associated with other structures. Column 1 gives the filament name, as in Li et al. (2016). Column 2 is the central velocity of ${ }^{13} \mathrm{CO}(2-1)$, measured in the SEDIGISM data; the velocity range used to compute column densities and masses is given in Col. 3, and the integrated intensity is in Col. 4. Column 5 is the projected area, computed for the assigned distance given in Col. 6. The total mass that we computed is given in Col. 7. Column 8 shows the length of each filament, and Col. 9 is the velocity dispersion. Finally, the measured and the virial linear mass densities are given in Cols. 10 and 11, respectively.


Fig. 26. ${ }^{13} \mathrm{CO}(2-1)$ (black) and $\mathrm{C}^{18} \mathrm{O}(2-1)$ (red) spectra extracted at six positions along the spine of the G341.246-0.267 filament (Fig. 25).

intensity maps of the ${ }^{13} \mathrm{CO}(2-1)$ and $\mathrm{C}^{18} \mathrm{O}(2-1)$ lines for each observed velocity component. We then compared the morphology of the molecular line emission with that of the dust emission to verify their association.

Eleven of the twelve ATLASGAL structures in the science demonstration field were detected in the SEDIGISM data. The undetected filament (G340.600+00.067) shows only weak dust emission and is located in the slightly noisier area of the field. Ten identified structures have a coherent velocity component along the spine (the main part of the skeleton as identified in ATLASGAL - see Fig. 25 for one example). The eleventh candidate (G340.630-00.093) shows several velocity components at all positions along the spine and the association with the dust structure is not clear. Therefore, we exclude this object from the current analysis. Another six structures (G340.482-00.306, G340.511-00.471, G340.981-00.013, G341.415+00.244, G340.236-00.153, G341.306+00.339) show additional velocity components which may contribute to the dust emission. One of them, G340.236-00.153, is defined as a network of filaments by $\mathrm{Li}$ et al. (2016) but splits into two networks of filaments at $-51.3 \mathrm{~km} \mathrm{~s}^{-1}$ and $-122.1 \mathrm{~km} \mathrm{~s}^{-1}$ in the ${ }^{13} \mathrm{CO}$ data. These are labelled as G340.236-00.153 and G340.200-00.035 in Table 6.

To estimate the mass of each filament, we computed the ${ }^{13} \mathrm{CO}$ column density at each pixel in the dilation box around the spine by integrating in velocity over a range equal to twice the average FWHM of the ${ }^{13} \mathrm{CO}$ component associated with the filament. Column densities were computed using the $X_{{ }^{13}} \mathrm{CO}(2-1)$ factor derived from Hi-GAL data (Sect. 4.4). We then computed the mass of each filament in its dilation box through the equation:

$M\left(\mathrm{H}_{2}\right)=\sum_{i} N_{i}\left(\mathrm{H}_{2}\right) A_{\mathrm{i}} \mu \mathrm{m}_{\mathrm{p}}$

where $N_{i}\left(\mathrm{H}_{2}\right)$ is the $\mathrm{H}_{2}$ column density computed for pixel $i, A_{i}$ its area, $\mu=2.8$ the mean molecular weight, and $m_{\mathrm{p}}$ the proton mass. We checked for contamination of other structures in the relevant velocity ranges and only in G340.301-00.387 and G340.482-00.306 there is contamination from structures at redshifted velocities from the main velocity of the filament up to $20 \%$ and $34 \%$, respectively.

The gravitational stability of a filament can be estimated by its linear mass density, or mass per unit length $(M / l)$. The virial linear mass density, above which a filament without additional support would collapse radially, is given by $(M / l)_{\mathrm{vir}}=$ $2 \sigma_{v}^{2} / G$, where $\sigma_{v}$ is the 1 -dimensional total (thermal plus non-thermal) velocity dispersion of the average molecular gas (Inutsuka \& Miyama 1997). Following Hatchell et al. (2005), 



Fig. 27. Left: spines of the $145 \mathrm{DisPerSE}$ filaments overlaid on the ${ }^{13} \mathrm{CO}$ integrated intensity map. The coloured lines correspond to the sub-sample of 33 filaments listed in Table B.1. Right: comparison between the ATLASGAL filaments (thick black lines) and the spines of the 145 DisPerSE structures (thin coloured lines).

we derived the velocity dispersion from the average FWHM of the line along the spine of each filament, excluding regions that show evidence of optically thick emission. The filamentary networks are too complex to assign a representative length. Therefore, the linear mass density was computed only for the seven single filaments detected in the field; the results are reported in Table 6.

Two filaments are clearly sub-critical, three are critical and two are just at the boundary, with $(M / l)_{\text {obs }} \approx(M / l)_{\text {vir. }}$. The ratio between the observed and virial mass per unit length, $(M / l)_{\mathrm{obs}} /(M / l)_{\mathrm{vir}}$, is ranging from 0.4 to 3.5 . This is somewhat high compared to results found by others (e.g. Hernandez \& Tan 2011; Contreras et al. 2013a), who found $(M / l)_{\mathrm{obs}} /(M / l)_{\mathrm{vir}}$ significantly less than unity in different filamentary structures. These studies concluded that filaments may not be gravitationally bound globally, although star formation is occurring in local regions. However, our measurements of masses include several uncertainties: the $X_{{ }^{13} \mathrm{CO}(2-1)}$ factor (Sect. 4.4), has an uncertainty of a factor of 2. Additionally, opacity effects were not taken into account, for which Hernandez \& Tan (2011) showed that optical depth correction factors can increase the column density by a factor 2 in the densest clumps embedded in IRDCs.

\subsection{Identification of filamentary structures on the SEDIGISM data}

The SEDIGISM data potentially includes more filaments than those identified in the previous section from the dust emission. Indeed, the velocity information contained in spectroscopic datasets automatically solves the problem of blending of unrelated structures along the line of sight, which can affect continuum surveys. Furthermore, it can reveal the presence of substructures with different velocities that might be not detectable in the continuum (e.g. Hacar et al. 2013; Henshaw et al. 2014). To investigate the potential of the SEDIGISM survey to study filaments, we applied the DisPerSE algorithm (Sousbie 2011) directly on the ${ }^{13} \mathrm{CO}(2-1)$ data cube of the science demonstration field. In the following, we briefly describe the methodology used and the main results; a more detailed description of the method will be given elsewhere (Suri et al., in prep.).

We applied the DisPerSE algorithm on a smoothed version of the ${ }^{13} \mathrm{CO}$ data cube, convolved to an angular resolution of $50^{\prime \prime}$ to improve the signal-to-noise ratio; the resulting $\mathrm{rms}$ is $\sim 0.5 \mathrm{~K}$.
DisPerSE first calculates a Morse-Smale complex (see Sousbie 2011) that consists of critical points, persistence pairs, filaments, walls and voids, with the most significant structures being associated with the highest persistence values. In our particular case, we used a detection threshold of 3- $\sigma(1.5 \mathrm{~K})$, and a persistence threshold of 3 , in order to select only the critical point pairs that stand out. With these criteria, DisPerSE creates a skeleton file that contains about 600 structures throughout the science demonstration field, including 145 composed of more than 15 pixels (i.e. more than three "beams" in the data cube smoothed to 50 " resolution). We will focus the rest of our analysis on these 145 filaments.

In the left panel of Fig. 27, we plot the skeleton of the 145 filaments identified by DisPerSE. Most filaments appear as substructures of the GMCs identified by SCIMES (Sect. 4). In the right panel of Fig. 27, we compare the filaments identified in ATLASGAL with the structures detected by DisPerSE. Overall, there is a good agreement between the filaments identified in the continuum and in the ${ }^{13} \mathrm{CO}(2-1)$ data cube, but DisPerSE finds a larger number of structures compared to ATLASGAL. Moreover, some filaments identified in the continuum seem to be composed of several substructures in the data cube. Thus, the search for filamentary structures directly on the data cube can reveal filaments that are not detected in continuum images, because of projection effects or sensitivity issues.

In the following we present a basic characterization of the metrics (projected length, width and aspect ratio) of the filaments identified by DisPerSE. The length of the filament is directly determined by adding the distance of consecutive skeleton points within a DisPerSE filament. The mean projected length of the filaments is about 8.1 arcmin, with a large dispersion ranging from $1 \mathrm{arcmin}$ to $50 \mathrm{arcmin}$. The width of each filament is determined from cuts perpendicular to the filament. A Gaussian function is fitted to the intensity profile along each cut, providing a measurement of the width at this point. The distribution of widths along a given filament is computed, and the mean value of this distribution is considered to be the width of the filament. The mean value and standard deviation for the widths of all 145 filaments is $2.2 \pm 1.1 \mathrm{arcmin}$. Finally, the aspect ratio is directly determined from the length-to-width ratio. The mean aspect ratio is 4.2, and ranges from 1 to 40 .

Out of the 145 DisPerSE structures characterised in this work, only 33 have an aspect ratio $>5$, and can, therefore, be 
considered as the best filament candidates. In Table B.1, we list their basic properties. Assuming distances to the filaments in the range 2 to $6 \mathrm{kpc}$ (as determined for GMCs in Sect. 4.3), the mean physical length and width are $4.7-14.1 \mathrm{pc}$ and $1.3-3.8 \mathrm{pc}$, respectively. The average width is much larger than the typical $0.1 \mathrm{pc}$ value measured towards nearby star forming regions (e.g. André et al. 2010), which can be explained by our angular resolution: a $50^{\prime \prime}$ (smoothed) beam corresponds to $0.5 \mathrm{pc}$ at a distance of $2 \mathrm{kpc}$. But similar widths (in the range 0.6-3.0 pc) were measured by Wang et al. (2015) for filaments located at 3-5 kpc. Finally, the lengths determined for the DisPerSE filaments are consistent with those derived in the previous section for the ATLASGAL filaments (see Table 6).

In summary, DisPerSE can be applied to the SEDIGISM data to search for filaments directly in $\ell b v$ data cubes. It is worth mentioning, however, that some of the structures identified by DisPerSE, with a relatively small aspect ratio, may correspond to fragments of longer structures, which might not be identified as single large-scale filaments because of intensity variations along the filament. A more detailed study of the spatial distribution, as well as the velocity relation between different DisPerSE structures, will be performed in forthcoming papers, after combining the data of all the SEDIGISM fields. This analysis may reveal large-scale filamentary structures, similar to the very long and thin IRDCs "Nessie" (Jackson et al. 2010; Goodman et al. 2014), the Snake (Wang et al. 2014) and many others (Wang et al. 2015).

\section{Conclusion and perspectives}

We have completed a molecular line survey of the southern Galactic plane, which covers $78 \mathrm{deg}^{2}$ in the ${ }^{13} \mathrm{CO}(2-1)$ and $\mathrm{C}^{18} \mathrm{O}(2-1)$ lines at an angular resolution of $30^{\prime \prime}$. The $1-\sigma$ sensitivity of $0.8 \mathrm{~K}$ at $0.25 \mathrm{~km} \mathrm{~s}^{-1}$ allows us to detect interstellar material down to a few $10^{21} \mathrm{~cm}^{-2}$ in $\mathrm{H}_{2}$ column density. This is well suited for mapping the structure of the Galactic ISM at an unprecedented level of detail, from the scale of giant molecular clouds and long filaments down to individual, dense molecular clumps. The pipeline processing of this massive data set (over $10^{7}$ independent spectra) is in progress.

In this first overview paper, we have focussed our analysis on a $1.5 \mathrm{deg}^{2}$ science demonstration field, to illustrate the potential of the survey. The main results can be summarised as follows:

1. Using the SCIMES algorithm, we have extracted 182 molecular clouds from the $l b v$ data cube within $1.5 \mathrm{deg}^{2}, 58$ of which are complexes with at least two sub-structures. In comparison, Rice et al. (2016) extracted 1064 molecular clouds in the full Galaxy using a similar technique applied to the allGalaxy CO survey of Dame et al. (2001); this demonstrates the power of our high-resolution data to provide a detailed view of the distribution of molecular clouds in the Milky Way.

2. We estimated the kinematical distances to all the molecular clouds and solved for the distance ambiguity thanks to the HISA method for the vast majority of them. The distance distribution of these 182 GMCs traces the spiral structure of the Galaxy, providing an accurate view on the position of the near Scutum-Centaurus arm, the near and far Norma arm, and the near and far $3 \mathrm{kpc}$ arm.

3. From an analysis of their virial parameters, we find that the molecular clouds are generally stable with a median virial parameter of $\sim 2$. However, the majority of the dense clumps within them (as traced by ATLASGAL) are unstable against gravitational collapse. Moreover, we observe a trend of decreasing virial parameters for increasing cloud and clump masses; the most massive clouds and clumps are the most gravitationally unstable.

4. Eighteen clumps (within 12 GMCs) are associated with massive star forming tracers and these tend to be the most massive and unstable of the 140 dense clumps located in the science demonstration field.

5. By combining the ${ }^{13} \mathrm{CO}(2-1)$ data of this survey with ${ }^{13} \mathrm{CO}(1-0)$ data from the ThrUMMS survey, we are able to solve the radiative transfer equations in order to compute excitation temperature, line opacity and column density in each voxel of the data cube.

6 . From the ratio of column density to integrated line intensity, we are also able to compute a ${ }^{13} \mathrm{CO}(2-1)$-to- $\mathrm{H}_{2} X$-factor of $1.0 \times 10^{21} \mathrm{~cm}^{-2}\left(\mathrm{~K} \mathrm{~km} \mathrm{~s}^{-1}\right)^{-1} \pm 0.2 \mathrm{dex}$. This is in excellent agreement with an independent estimate of this factor based on the comparison between an $\mathrm{H}_{2}$ column density map derived from Hi-GAL data and the ${ }^{13} \mathrm{CO}(2-1)$ SEDIGISM data.

7. Most of the filaments previously extracted from continuum surveys (ATLASGAL, Hi-GAL) are detected in ${ }^{13} \mathrm{CO}$. The velocity information allows us to confirm that they are coherent structures in $\sim 80 \%$ cases.

8. Using the DisPerSE algorithm directly on the ${ }^{13} \mathrm{CO}$ data, we extracted 145 filamentary structures with lengths above $150^{\prime \prime}, 33$ of which have an aspect ratio greater than 5 .

The science demonstration field covers only $\sim 2 \%$ of the full survey area. Therefore, we can expect to build catalogues with several $10^{4}$ molecular clouds, and several $10^{3}$ filaments from the entire survey data; we will also be able to assign distances and investigate their Galactic distribution. We may detect filamentary structures on degree-scales, which would be of prime importance to constrain the formation mechanism of filaments in the ISM. This unique data set will also allow us to put strong constraints on the star-formation efficiency as a function of environment.

Finally, the SEDIGISM survey in the $1 \mathrm{~mm}$ band is well complemented with the ThrUMMS survey at $3 \mathrm{~mm}$ wavelength, and with other, ongoing surveys in higher $J$ transitions. Combining these data sets allows us to study in $3 \mathrm{D}$ the excitation conditions in the Galactic ISM, at $\lesssim 1^{\prime}$ resolution, and to put strong constraints on the combination of excitation temperature $\left(T_{\mathrm{ex}}\right)$, optical depth $(\tau)$, and the $\mathrm{CO}-$ to- $\mathrm{H}_{2}$ conversion factor. When also considering the data from the GRS in the first quadrant, this will provide us for the first time with a global, yet detailed view of the bulk of the interstellar matter in the inner Galaxy.

The SEDIGISM data products (calibrated data cubes, catalogues of clouds and filaments) will be made public shortly after being processed and the quality has been carefully checked. This will give this survey a high legacy value for Milky Way studies in the southern hemisphere.

Acknowledgements. We thank the anonymous referee for his/her constructive comments, which helped us in improving the clarity of the text. This document was produced using the Overleaf web application, which can be found at www . overleaf.com. This work has used the CAMELOT project (Ginsburg et al. 2016); http://camelot-project.org. We would like to thank David Eden for useful discussions about the dense gas fraction. We are very grateful to Dario Colombo for fruitful discussions and interactions regarding the use of the SCIMES software. T.Cs. and K.W. are supported by the Deutsche Forschungsgemeinschaft (DFG) priority programme 1573 ISM-SPP. A.S.M., P.S., and S.S. are partially supported by the DFG through grant SFB 956, subprojects A4 and A6. A.D.C. and C.L.D. acknowledge funding from the European Research Council for the FP7 ERC starting grant project LOCALSTAR. L.B. acknowledges 
support by CONICYT grant PFB-06. P.J.B. acknowledges grant support from NSF AST-1312597 and NASA-ADAP NNX15AF64G.

\section{References}

Abreu-Vicente, J., Ragan, S., Kainulainen, J., et al. 2016, A\&A, 590, A131 Aguirre, J. E., Ginsburg, A. G., Dunham, M. K., et al. 2011, ApJS, 192, 4 Anderson, L. D., \& Bania, T. M. 2009, ApJ, 690, 706 André, P., Men'shchikov, A., Bontemps, S., et al. 2010, A\&A, 518, L102 Barnes, P. J., Yonekura, Y., Ryder, S. D., et al. 2010, MNRAS, 402, 73 Barnes, P. J., Yonekura, Y., Fukui, Y., et al. 2011, ApJS, 196, 12 Barnes, P. J., Muller, E., Indermuehle, B., et al. 2015, ApJ, 812, 6

Barnes, P. J., Hernandez, A. K., O’Dougherty, S. N., Schap, III, W. J., \& Muller, E. 2016, ApJ, 831, 67

Battersby, C., Ginsburg, A., Bally, J., et al. 2014, ApJ, 787, 113

Battisti, A. J., \& Heyer, M. H. 2014, ApJ, 780, 173

Benjamin, R. A., Churchwell, E., Babler, B. L., et al. 2003, PASP, 115, 953

Bertoldi, F., \& McKee, C. F. 1992, ApJ, 395, 140

Bihr, S., Johnston, K. G., Beuther, H., et al. 2016, A\&A, 588, A97

Bolatto, A. D., Leroy, A. K., Rosolowsky, E., Walter, F., \& Blitz, L. 2008, ApJ, 686, 948

Bolatto, A. D., Wolfire, M., \& Leroy, A. K. 2013, ARA\&A, 51, 207

Braiding, C., Burton, M. G., Blackwell, R., et al. 2015, PASA, 32, 20

Brand, J., \& Blitz, L. 1993, A\&A, 275, 67

Bronfman, L., Cohen, R. S., Alvarez, H., May, J., \& Thaddeus, P. 1988, ApJ, 324, 248

Brunt, C. M., \& Heyer, M. H. 2002a, ApJ, 566, 276

Brunt, C. M., \& Heyer, M. H. 2002b, ApJ, 566, 289

Brunt, C. M., \& Heyer, M. H. 2013, MNRAS, 433, 117

Brunt, C. M., \& Mac Low, M.-M. 2004, ApJ, 604, 196

Brunt, C. M., Heyer, M. H., Vázquez-Semadeni, E., \& Pichardo, B. 2003, ApJ, 595,824

Burgers, J. M. 1974, The Nonlinear Diffusion Equation (The Netherlands: Springer)

Burkhart, B., Lazarian, A., Ossenkopf, V., \& Stutzki, J. 2013, ApJ, 771, 123

Burton, M. G., Braiding, C., Glueck, C., et al. 2013, PASA, 30, 44

Busfield, A. L., Purcell, C. R., Hoare, M. G., et al. 2006, MNRAS, 366, 1096

Carey, S. J., Noriega-Crespo, A., Mizuno, D. R., et al. 2009, PASP, 121, 76

Churchwell, E., Babler, B. L., Meade, M. R., et al. 2009, PASP, 121, 213

Colombo, D., Hughes, A., Schinnerer, E., et al. 2014, ApJ, 784, 3

Colombo, D., Rosolowsky, E., Ginsburg, A., Duarte-Cabral, A., \& Hughes, A. 2015, MNRAS, 454, 2067

Contreras, Y., Rathborne, J., \& Garay, G. 2013a, MNRAS, 433, 251

Contreras, Y., Schuller, F., Urquhart, J. S., et al. 2013b, A\&A, 549, A45

Csengeri, T., Urquhart, J. S., Schuller, F., et al. 2014, A\&A, 565, A75

Csengeri, T., Weiss, A., Wyrowski, F., et al. 2016, A\&A, 585, A104

Dame, T. M., Hartmann, D., \& Thaddeus, P. 2001, ApJ, 547, 792

Deharveng, L., Zavagno, A., Samal, M. R., et al. 2015, A\&A, 582, A1

Dempsey, J. T., Thomas, H. S., \& Currie, M. J. 2013, ApJS, 209, 8

Dickey, J. M., McClure-Griffiths, N. M., Stanimirović, S., Gaensler, B. M., \& Green, A. J. 2001, ApJ, 561, 264

Dobbs, C. L. 2015, MNRAS, 447, 3390

Dobbs, C. L., Burkert, A., \& Pringle, J. E. 2011, MNRAS, 413, 2935

Dobbs, C. L., Krumholz, M. R., Ballesteros-Paredes, J., et al. 2014, Protostars and Planets VI, 3

Duarte-Cabral, A., \& Dobbs, C. L. 2016, MNRAS, 458, 3667

Dunham, M. K., Rosolowsky, E., Evans, II, N. J., Cyganowski, C., \& Urquhart, J. S. 2011, ApJ, 741, 110

Eden, D. J., Moore, T. J. T., Morgan, L. K., Thompson, M. A., \& Urquhart, J. S. 2013, MNRAS, 431, 1587

Elia, D., Molinari, S., Fukui, Y., et al. 2013, ApJ, 772, 45

Elmegreen, B. G., \& Scalo, J. 2004, ARA\&A, 42, 211

Esquivel, A., Lazarian, A., Pogosyan, D., \& Cho, J. 2003, MNRAS, 342, 325

Federrath, C. 2016, MNRAS, 457, 375

García, P., Bronfman, L., Nyman, L.-̊̊., Dame, T. M., \& Luna, A. 2014, ApJS, 212, 2

Giannetti, A., Brand, J., Sánchez-Monge, Á., et al. 2013, A\&A, 556, A16

Ginsburg, A., Bressert, E., Bally, J., \& Battersby, C. 2012, ApJ, 758, L29

Ginsburg, A., Kruijssen, J., Longmore, S., et al. 2016, Astrophysics Source Code Library [record ascl: 1605.006]

Goodman, A. A., Alves, J., Beaumont, C. N., et al. 2014, ApJ, 797, 53

Güsten, R., Nyman, L. A., Schilke, P., et al. 2006, A\&A, 454, L13

Gutermuth, R. A., \& Heyer, M. 2015, AJ, 149, 64

Guzmán, A. E., Sanhueza, P., Contreras, Y., et al. 2015, ApJ, 815, 130

Hacar, A., Tafalla, M., Kauffmann, J., \& Kovács, A. 2013, A\&A, 554, A55

Hatchell, J., Richer, J. S., Fuller, G. A., et al. 2005, A\&A, 440, 151

Heitsch, F., Hartmann, L. W., Slyz, A. D., Devriendt, J. E. G., \& Burkert, A. 2008, ApJ, 674, 316
Henshaw, J. D., Caselli, P., Fontani, F., Jiménez-Serra, I., \& Tan, J. C. 2014, MNRAS, 440, 2860

Hernandez, A. K., \& Tan, J. C. 2011, ApJ, 730, 44

Hernandez, A. K., Tan, J. C., Caselli, P., et al. 2011, ApJ, 738, 11

Heyer, M., \& Dame, T. M. 2015, ARA\&A, 53, 583

Heyer, M., Krawczyk, C., Duval, J., \& Jackson, J. M. 2009, ApJ, 699, 1092

Hildebrand, R. H. 1983, QJRAS, 24, 267

Hill, T., Burton, M. G., Minier, V., et al. 2005, MNRAS, 363, 405

Hoare, M. G., Purcell, C. R., Churchwell, E. B., et al. 2012, PASP, 124, 939

Inutsuka, S.-I., \& Miyama, S. M. 1997, ApJ, 480, 681

Jackson, J. M., Bania, T. M., Simon, R., et al. 2002, ApJ, 566, L81

Jackson, J. M., Rathborne, J. M., Shah, R. Y., et al. 2006, ApJS, 163, 145

Jackson, J. M., Finn, S. C., Chambers, E. T., Rathborne, J. M., \& Simon, R. 2010, ApJ, 719, L185

Jackson, J. M., Rathborne, J. M., Foster, J. B., et al. 2013, PASA, 30, e057

Jiménez-Serra, I., Caselli, P., Tan, J. C., et al. 2010, MNRAS, 406, 187

Kauffmann, J., Pillai, T., \& Goldsmith, P. F. 2013, ApJ, 779, 185

Khoperskov, S. A., Vasiliev, E. O., Sobolev, A. M., \& Khoperskov, A. V. 2013, MNRAS, 428, 2311

Klein, B., Hochgürtel, S., Krämer, I., et al. 2012, A\&A, 542, L3

Kolmogorov, A. 1941, Akademiia Nauk SSSR Doklady, 30, 301

Kramer, C., Alves, J., Lada, C. J., et al. 1999, A\&A, 342, 257

Lada, C. J., Lombardi, M., \& Alves, J. F. 2010, ApJ, 724, 687

Langer, W. D., Velusamy, T., Pineda, J. L., Willacy, K., \& Goldsmith, P. F. 2014, A\&A, 561, A122

Lazarian, A., \& Pogosyan, D. 2000, ApJ, 537, 720

Levine, E. S., Blitz, L., \& Heiles, C. 2006, Science, 312, 1773

Li, G.-X., Urquhart, J. S., Leurini, S., et al. 2016, A\&A, 591, A5

Marshall, D. J., Robin, A. C., Reylé, C., Schultheis, M., \& Picaud, S. 2006, A\&A, 453,635

McClure-Griffiths, N. M., \& Dickey, J. M. 2007, ApJ, 671, 427

McClure-Griffiths, N. M., Dickey, J. M., Gaensler, B. M., et al. 2005, ApJS, 158, 178

McKee, C. F., \& Ostriker, E. C. 2007, ARA\&A, 45, 565

Medina, S.-N. X., Arthur, S. J., Henney, W. J., Mellema, G., \& Gazol, A. 2014, MNRAS, 445, 1797

Molinari, S., Swinyard, B., Bally, J., et al. 2010, A\&A, 518, L100

Motte, F., Bontemps, S., Schilke, P., et al. 2007, A\&A, 476, 1243

Mulder, W. A., \& Liem, B. T. 1986, A\&A, 157, 148

Muller, E., Stanimirović, S., Rosolowsky, E., \& Staveley-Smith, L. 2004, ApJ, 616,845

Nagai, T., Inutsuka, S.-I., \& Miyama, S. M. 1998, ApJ, 506, 306

Nakamura, F., \& Li, Z.-Y. 2008, ApJ, 687, 354

Narayanan, D., Krumholz, M. R., Ostriker, E. C., \& Hernquist, L. 2012, MNRAS, 421, 3127

Peretto, N., \& Fuller, G. A. 2009, A\&A, 505, 405

Peretto, N., Fuller, G. A., Duarte-Cabral, A., et al. 2013, A\&A, 555, A112

Pettitt, A. R., Dobbs, C. L., Acreman, D. M., \& Price, D. J. 2014, MNRAS, 444, 919

Pettitt, A. R., Dobbs, C. L., Acreman, D. M., \& Bate, M. R. 2015, MNRAS, 449, 3911

Pillai, T., Kauffmann, J., Tan, J. C., et al. 2015, ApJ, 799, 74

Ragan, S. E., Henning, T., Tackenberg, J., et al. 2014, A\&A, 568, A73

Rathborne, J. M., Johnson, A. M., Jackson, J. M., Shah, R. Y., \& Simon, R. 2009, ApJS, 182, 131

Rice, T. S., Goodman, A. A., Bergin, E. A., Beaumont, C., \& Dame, T. M. 2016, ApJ, 822, 52

Rigby, A. J., Moore, T. J. T., Plume, R., et al. 2016, MNRAS, 456, 2885

Roman-Duval, J., Jackson, J. M., Heyer, M., et al. 2009, ApJ, 699, 1153

Roman-Duval, J., Federrath, C., Brunt, C., et al. 2011, ApJ, 740, 120

Rosolowsky, E. 2007, ApJ, 654, 240

Rosolowsky, E., Engargiola, G., Plambeck, R., \& Blitz, L. 2003, ApJ, 599, 258

Rosolowsky, E. W., Pineda, J. E., Kauffmann, J., \& Goodman, A. A. 2008, ApJ, 679,1338

Russeil, D. 2003, A\&A, 397, 133

Santangelo, G., Testi, L., Leurini, S., et al. 2010, A\&A, 520, A50

Schisano, E., Rygl, K. L. J., Molinari, S., et al. 2014, ApJ, 791, 27

Schneider, N., Csengeri, T., Bontemps, S., et al. 2010, A\&A, 520, A49

Schuller, F., Menten, K. M., Contreras, Y., et al. 2009, A\&A, 504, 415

Smith, R. J., Glover, S. C. O., Klessen, R. S., \& Fuller, G. A. 2016, MNRAS, 455,3640

Sousbie, T. 2011, MNRAS, 414, 350

Taylor, J. H., \& Cordes, J. M. 1993, ApJ, 411, 674

Urquhart, J. S., Busfield, A. L., Hoare, M. G., et al. 2007, A\&A, 474, 891

Urquhart, J. S., Morgan, L. K., Figura, C. C., et al. 2011, MNRAS, 418, 1689

Urquhart, J. S., Moore, T. J. T., Schuller, F., et al. 2013, MNRAS, 431, 1752

Urquhart, J. S., Csengeri, T., Wyrowski, F., et al. 2014a, A\&A, 568, A41

Urquhart, J. S., Figura, C. C., Moore, T. J. T., et al. 2014b, MNRAS, 437, 1791 
Urquhart, J. S., Moore, T. J. T., Csengeri, T., et al. 2014c, MNRAS, 443, 1555 Urquhart, J. S., Figura, C. C., Moore, T. J. T., et al. 2015, MNRAS, 452, 4029 van der Tak, F. F. S., Black, J. H., Schöier, F. L., Jansen, D. J., \& van Dishoeck, E. F. 2007, A\&A, 468, 627

Vassilev, V., Meledin, D., Lapkin, I., et al. 2008, A\&A, 490, 1157

Walker, D. L., Longmore, S. N., Bastian, N., et al. 2015, MNRAS, 449, 715

Walsh, A. J., Breen, S. L., Britton, T., et al. 2011, MNRAS, 416, 1764

Wang, K., Wu, Y. F., Ran, L., Yu, W. T., \& Miller, M. 2009, A\&A, 507, 369

Wang, K., Zhang, Q., Testi, L., et al. 2014, MNRAS, 439, 3275

Wang, K., Testi, L., Ginsburg, A., et al. 2015, MNRAS, 450, 4043

Wei, L. H., Keto, E., \& Ho, L. C. 2012, ApJ, 750, 136

Wienen, M., Wyrowski, F., Schuller, F., et al. 2012, A\&A, 544, A146

Wienen, M., Wyrowski, F., Menten, K. M., et al. 2015, A\&A, 579, A91
Wong, T., Hughes, A., Ott, J., et al. 2011, ApJS, 197, 16

Wright, E. L., Eisenhardt, P. R. M. Mainzer, A. K., et al. 2010, AJ, 140, 1868

Zucker, C., Battersby, C., \& Goodman, A. 2015, ApJ, 815, 23

\section{Appendix A: Full catalogue of clouds} in the SEDIGISM science demonstration field

The properties of all clouds from the SCIMES extraction (Table A.1) are only available at the CDS.

\section{Appendix B: Properties of the DisPerSE filaments}

Table B.1. Properties of the 33 DisPerSE filaments with aspect ratios $>5$. Columns 2 to 5 give the positions of the extrema (minimum and maximum in Galactic coordinates). Columns 6 and 7 indicate the projected length and width; the aspect ratio is given in Col. 8.

\begin{tabular}{|c|c|c|c|c|c|c|c|}
\hline Name & $g l 1$ & $g b 1$ & $g l 2$ & $\underset{\circ}{g b 2}$ & $\begin{array}{l}\text { Length } \\
\text { (arcmin) }\end{array}$ & $\begin{array}{c}\text { Width } \\
\text { (arcmin) }\end{array}$ & $R$ \\
\hline Dis001 & 341.4252 & +0.3797 & 341.3725 & -0.0346 & 48.85 & 1.27 & 38.6 \\
\hline Dis002 & 340.9872 & -0.0557 & 340.5834 & -0.0029 & 43.08 & 1.18 & 36.4 \\
\hline Dis003 & 340.5043 & -0.2404 & 340.2457 & -0.0478 & 25.98 & 0.81 & 32.2 \\
\hline Dis004 & 340.3749 & -0.3565 & 340.0398 & -0.4172 & 28.93 & 1.70 & 17.0 \\
\hline Dis005 & 341.0901 & -0.0161 & 340.9318 & -0.2404 & 35.07 & 2.19 & 16.0 \\
\hline Dis006 & 341.0162 & -0.1032 & 340.6995 & -0.2299 & 40.39 & 2.88 & 14.0 \\
\hline Dis007 & 340.8051 & +0.1950 & 340.8473 & +0.2003 & 13.50 & 0.96 & 14.0 \\
\hline Dis008 & 341.2273 & -0.5096 & 341.3514 & -0.3038 & 27.86 & 2.15 & 13.0 \\
\hline Dis009 & 341.2062 & +0.0736 & 341.3012 & +0.0314 & 10.96 & 0.88 & 12.4 \\
\hline Dis010 & 340.5834 & -0.1507 & 340.2536 & -0.0478 & 36.53 & 2.83 & 12.9 \\
\hline Dis011 & 340.6573 & -0.0927 & 340.5333 & -0.1428 & 27.58 & 2.62 & 10.5 \\
\hline Dis012 & 341.3962 & +0.0314 & 341.2036 & +0.0841 & 20.11 & 1.91 & 10.5 \\
\hline Dis013 & 340.6995 & -0.2325 & 340.6468 & -0.1006 & 15.65 & 1.62 & 9.7 \\
\hline Dis014 & 340.7708 & +0.3085 & 340.5729 & +0.3612 & 17.30 & 1.78 & 9.7 \\
\hline Dis015 & 340.1005 & -0.3170 & 340.0636 & -0.1692 & 12.20 & 1.35 & 9.1 \\
\hline Dis016 & 340.2245 & -0.3090 & 340.0081 & -0.1507 & 22.71 & 2.53 & 9.0 \\
\hline Dis017 & 340.2245 & -0.1560 & 340.2984 & -0.2220 & 25.69 & 2.92 & 8.8 \\
\hline Dis018 & 340.5254 & -0.0900 & 340.4040 & -0.1164 & 11.41 & 1.56 & 7.3 \\
\hline Dis019 & 341.4595 & +0.3190 & 341.2352 & +0.3348 & 21.29 & 2.94 & 7.2 \\
\hline Dis020 & 340.5861 & -0.3196 & 340.5254 & -0.2642 & 6.18 & 0.86 & 7.2 \\
\hline Dis021 & 341.5097 & +0.2478 & 341.5070 & +0.2398 & 5.22 & 0.74 & 7.1 \\
\hline Dis022 & 341.0769 & -0.3513 & 340.9344 & -0.2325 & 17.41 & 2.56 & 6.8 \\
\hline Dis023 & 341.4648 & +0.2847 & 341.3725 & +0.1660 & 16.94 & 2.59 & 6.5 \\
\hline Dis024 & 341.1904 & -0.1164 & 341.1138 & -0.1296 & 5.71 & 0.91 & 6.3 \\
\hline Dis025 & 341.0109 & -0.0003 & 340.9661 & +0.0155 & 5.33 & 0.89 & 6.0 \\
\hline Dis026 & 340.4673 & -0.3988 & 340.2456 & -0.3724 & 24.93 & 4.40 & 5.7 \\
\hline Dis027 & 341.0980 & -0.3302 & 341.0241 & -0.3803 & 7.72 & 1.37 & 5.6 \\
\hline Dis028 & 340.9397 & -0.0056 & 340.9212 & +0.1158 & 9.66 & 1.80 & 5.4 \\
\hline Dis029 & 340.5306 & -0.1428 & 340.4066 & -0.0742 & 13.91 & 2.65 & 5.2 \\
\hline Dis030 & 340.1322 & +0.0947 & 340.0372 & +0.0947 & 11.27 & 2.21 & 5.1 \\
\hline Dis031 & 341.2484 & -0.4040 & 341.1693 & -0.2906 & 12.49 & 2.51 & 5.0 \\
\hline Dis032 & 340.2984 & -0.3275 & 340.2193 & -0.2272 & 10.87 & 2.17 & 5.0 \\
\hline Dis033 & 340.2061 & +0.1000 & 340.1375 & +0.1369 & 6.28 & 1.26 & 5.0 \\
\hline
\end{tabular}

